\title{
Spliceator: multi-species splice site prediction using convolutional neural networks
}

\author{
Nicolas Scalzitti , Arnaud Kress ${ }^{1,2}$, Romain Orhand ${ }^{1}$, Thomas Weber ${ }^{1}$, Luc Moulinier ${ }^{1,2}$, Anne Jeannin-Girardon ${ }^{1}$, \\ Pierre Collet ${ }^{1}$, Olivier Poch ${ }^{1}$ and Julie D. Thompson ${ }^{1 *}$ (1)
}

\author{
*Correspondence: \\ thompson@unistra.fr \\ ${ }^{1}$ Complex Systems \\ and Translational \\ Bioinformatics (CSTB), ICube \\ Laboratory, UMR7357, \\ University of Strasbourg, \\ 1 rue Eugène Boeckel, \\ 67000 Strasbourg, France \\ Full list of author information \\ is available at the end of the \\ article
}

\begin{abstract}
Background: Ab initio prediction of splice sites is an essential step in eukaryotic genome annotation. Recent predictors have exploited Deep Learning algorithms and reliable gene structures from model organisms. However, Deep Learning methods for non-model organisms are lacking.

Results: We developed Spliceator to predict splice sites in a wide range of species, including model and non-model organisms. Spliceator uses a convolutional neural network and is trained on carefully validated data from over 100 organisms. We show that Spliceator achieves consistently high accuracy (89-92\%) compared to existing methods on independent benchmarks from human, fish, fly, worm, plant and protist organisms.
\end{abstract}

Conclusions: Spliceator is a new Deep Learning method trained on high-quality data, which can be used to predict splice sites in diverse organisms, ranging from human to protists, with consistently high accuracy.

Keywords: Splice site prediction, Genome annotation, Data quality, Deep learning, Convolutional neural network

\section{Background}

The raw genomic sequences generated by next generation sequencing (NGS) are an important source of data for studying and understanding organisms and biological mechanisms. However, without a crucial step of extracting biological knowledge from the raw data, a process called 'genome annotation', the sequences are difficult to exploit and may even be useless. A critical step in the annotation process involves the location of genes (i.e. 'structural annotation'), in particular the protein-coding genes and the characterization of their intron/exon structures. A large number of automatic annotation pipelines have been developed to identify protein-coding genes, such as Braker2 [1], Maker [2] or PASA [3], as well as dedicated resources, such as Ensembl [4] or NCBI [5]. Automatic annotation methods are generally based on a combination of empirical evidence, e.g. mRNA sequencing (RNA-seq) data or known gene structures from closely related organisms, and ab initio gene prediction programs, such as Augustus [6], author(s) and the source, provide a link to the Creative Commons licence, and indicate if changes were made. The images or other third party material in this article are included in the article's Creative Commons licence, unless indicated otherwise in a credit line to the material. If material is not included in the article's Creative Commons licence and your intended use is not permitted by statutory regulation or exceeds the permitted use, you will need to obtain permission directly from the copyright holder. To view a copy of this licence, visit http:// creativecommons.org/licenses/by/4.0/. The Creative Commons Public Domain Dedication waiver (http://creativecommons.org/publi cdomain/zero/1.0/) applies to the data made available in this article, unless otherwise stated in a credit line to the data. 
Genscan [7], Snap [8] or GlimmerHMM [9]. Despite these developments, the annotation of gene structure remains a major challenge, especially for eukaryotic organisms [10-13] due to their complex exon-intron mosaics [14] (Fig. 1).

Eukaryotic gene prediction involves determining the internal architecture of each gene, including the start and stop codons, and the boundaries between each exon and intron, called splice sites (SS). The SS are specifically recognized by the spliceosome, a ribonucleoprotein complex [15], and play an important role in the diversity of the proteome $[16,17]$. There are two types of SS, the 5 ' site (also called donor site) and the 3' site (or acceptor site), located respectively at the exon-intron and intron-exon junctions. SS are generally characterized by the presence of the dinucleotide GT at the 5' site and AG at the 3' site, called canonical sites [18]. The dinucleotides are embedded in longer, consensus motifs: aGGTAAGT (Donor) and (Y)6N(C/t)AG(g/a)t (Acceptor) [19]. Although the canonical SS are highly conserved [20] and represent more than $98.3 \%$ of SS in animals, $98.7 \%$ in fungi and $97.9 \%$ in plants [21], there are some exceptions, such as the presence of the dinucleotides AT-AC or GC-AG [22, 23], described as non-canonical sites. Thus, the challenges in accurately predicting all SS in a genome are twofold. First, the huge number of GT and AG dinucleotides that are not located at SS can generate a high rate of false positives. Second, the presence of non-canonical SS can lead to false negative predictions if they are not taken into account [24].

A number of methods have been developed to identify SS by exploiting recent highthroughput RNA-seq data, for instance MapSplice [25], TopHat [26] or SplitSeek [27]. However, this approach depends on the availability of high quality data and a minimal depth of sequencing to be able to detect all SS, in particular those in low-expressed isoforms [28]. As a consequence, alternative approaches are needed to identify SS based solely on the genome sequence. Most of them exploit machine learning (ML) algorithms and use several features to describe SS, covering the consensus motifs or other nucleotides in proximity to the SS [29]. The most widely used ML algorithms include Support Vector Machines [30-32], Markov models [33, 34], Random Forest [35, 36] and Bayesian networks [37]. However, these methods are limited by the lack of knowledge about the input sequence (patterns, secondary structures, etc.), complex biological processes [38], a weak genomic context (the region around the SS) and the construction and selection of pertinent feature sets [29], which is often time-consuming. More recently, programs using deep learning (DL) algorithms have been introduced, such as DSSP [39],

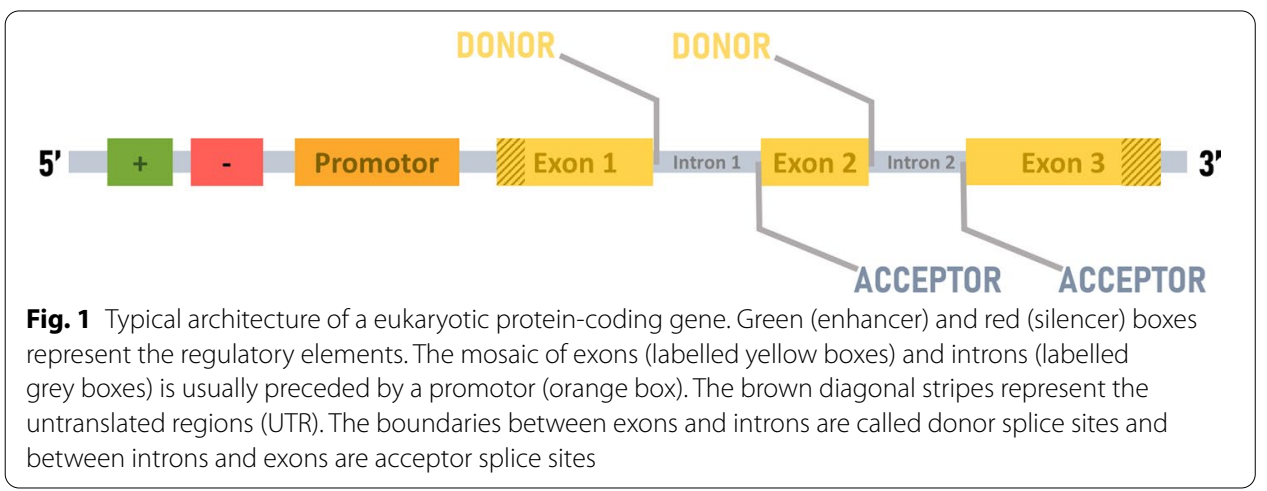


SpliceRover [40], SpliceFinder [41], or SpliceAI [42]. The DL approaches are based on convolutional neural networks $(\mathrm{CNN})$ and do not require the manual definition of a feature set, because they automatically extract the most pertinent characteristics to classify elements (here, splice sites) in different classes determined by the initial problem [43]. Another advantage of these algorithms is that they are able to find correlations between features in a larger region (i.e. in the genomic sequence). In the context of SS detection, this characteristic is important, as several elements are involved, such as the branchpoint site (BPS), intronic splicing enhancers (ISE), intronic splicing silencers (ISS), exonic splicing enhancers (ESE) and exonic splicing silencers (ESS). Moreover, CNN use fewer parameters than classical multi-layer perceptrons, reducing the risk of overfitting [44], and they also share these parameters to extract local features.

DL methods rely on the availability of high quality data that is pertinent to the problem being solved, in order to train accurate models. For this reason, most of the current SS predictors have been trained on data restricted to humans or other model organisms. To our knowledge, there are no SS prediction tools trained on data from a large range of less well studied organisms, such as insects (except fruit fly), fungi or protists.

In this context, we have developed Spliceator, a new tool for ab initio prediction of eukaryotic multi-species splice sites. Spliceator is based on the CNN technology and more importantly, is trained on an original high quality dataset [45] containing genomic sequences from organisms ranging from human to protists. The training dataset has been rigorously established and validated to reduce the number of errors in the input data and avoid introducing bias in the learning process. This dataset allows us to limit the 'garbage-in, garbage-out' effect [46], meaning that poor quality data lead to less reliable results. Based on several benchmark experiments, we show that Spliceator achieves overall high accuracy compared to other state-of-the-art programs, including the neural network-based NNSplice [47], MaxEntScan [48] that models SS using the maximum entropy distribution, and two CNN-based methods: DSSP [39] and SpliceFinder [41]. More importantly, Spliceator performance is robust and remains consistently high for sequences from diverse organisms ranging from human to protists.

\section{Results}

\section{Design of training and test datasets for multi-species SS prediction}

Since we employ a supervised learning approach, the careful construction of the positive and negative datasets used for training the $\mathrm{CNN}$ models is essential. We designed eight strategies to build different datasets, where each strategy highlights a parameter that can influence the model performance, such as the input sequence length, the data quality, the type of negative sequences (only false positives (FP) or exon, intron and FP sequences) and the dataset composition, i.e. the effect of balanced or unbalanced datasets with different ratios between the number of positive and negative sequences. Each dataset was then split into separate training and test sets in order to build prediction models for donor and acceptor SS using CNN.

The first dataset, called All Sequences (AS), includes sequences from the 1361 'Confirmed' (error-free) gene sequences available in the G3PO+ dataset (see "Methods" section), as well as the 1380 'Unconfirmed' sequences that contain potential gene prediction errors. The AS dataset is designed to represent real-world problems, in the sense that 
the data is extracted directly from public databases. For the second dataset, called Gold Standard (GS), we exploited only the 'Confirmed' sequences implying that this set is error-free. The resulting AS and GS positive subsets include donor and acceptor SS from human, as well as from a diverse set of 147 eukaryotic organisms, ranging from primates to protists. In order to construct a robust negative subset of non-SS sequences, we again exploited both 'Confirmed' and 'Unconfirmed' sequences for the AS dataset and only 'Confirmed' sequences for the GS dataset. To do this, we randomly selected sequences from the exon/intron regions of the G3PO+ genomic sequences, as well as sequences containing GT/AG dinucleotides that are not SS.

In order to verify that there is no over-representation of sequences from specific organisms or clades, we calculated the mean pairwise percent identity for the input sequences with a length of 600 nucleotides (nt) (Table 1) and showed that the majority (>90\%) of the sequences in each positive subset (AS and GS) for both donor and acceptor SS share between 20 and 30\% identity. We also calculated the mean pairwise percent identity for sequences with a length of 20 nt, i.e. specifically the short region around SS. Again, the pairwise percent identity is similar for AS and GS positive datasets, however the majority of these sequences share between 20 and $60 \%$, showing that the context close to the SS is more conserved. Interestingly, the donor sequences of length $20 \mathrm{nt}$ are more conserved than acceptor sequences, e.g. in the GS dataset, $37.17 \%$ of donor sequences share $40-50 \%$ mean identity compared to $34.09 \%$ of acceptor sequences.

\section{Impact of genomic context}

To evaluate the impact of the genomic context around the SS on the prediction performance of our CNN method, we constructed subsets of sequences for the AS and GS datasets having different lengths, ranging from 20 to $600 \mathrm{nt}$. The sequence segments upstream and downstream of the SS dinucleotide contain information allowing the discrimination of SS and non-SS, such as the BPS, polypyrimidine tract (PPT) or regulatory cis-elements including exon/intron splicing enhancers or silencers (ESE/ISE or ESS/ISS)

Table 1 Distribution of sequences according to the mean percent identity

\begin{tabular}{|c|c|c|c|c|c|c|c|c|}
\hline \multirow{3}{*}{$\begin{array}{l}\text { Pairwise } \\
\text { sequence } \\
\text { identity }\end{array}$} & \multicolumn{4}{|c|}{ Sequence length: $600 \mathrm{nt}$} & \multicolumn{4}{|c|}{ Sequence length: $20 \mathrm{nt}$} \\
\hline & \multicolumn{2}{|l|}{ Donor } & \multicolumn{2}{|c|}{ Acceptor } & \multicolumn{2}{|l|}{ Donor } & \multicolumn{2}{|c|}{ Acceptor } \\
\hline & AS & GS & AS & GS & AS & GS & AS & GS \\
\hline $0-10 \%$ & $0.0 \%$ & $0.0 \%$ & $0.0 \%$ & $0.0 \%$ & $0.04 \%$ & $0.03 \%$ & $0.04 \%$ & $0.03 \%$ \\
\hline $10-20 \%$ & $0.35 \%$ & $0.27 \%$ & $0.33 \%$ & $0.25 \%$ & $1.0 \%$ & $0.71 \%$ & $1.65 \%$ & $1.32 \%$ \\
\hline $20-30 \%$ & $92.77 \%$ & $94.88 \%$ & $92.45 \%$ & $94.7 \%$ & $10.27 \%$ & $8.93 \%$ & $13.92 \%$ & $12.44 \%$ \\
\hline $30-40 \%$ & $6.83 \%$ & $4.78 \%$ & $7.18 \%$ & $4.99 \%$ & $32.62 \%$ & $31.57 \%$ & $34.05 \%$ & $33.20 \%$ \\
\hline $40-50 \%$ & $0.03 \%$ & $0.04 \%$ & $0.03 \%$ & $0.04 \%$ & $36.24 \%$ & $37.17 \%$ & $32.89 \%$ & $34.09 \%$ \\
\hline $50-60 \%$ & $0.01 \%$ & $0.01 \%$ & $0.01 \%$ & $0.01 \%$ & $16.28 \%$ & $17.47 \%$ & $14.22 \%$ & $15.34 \%$ \\
\hline $60-70 \%$ & $0.0 \%$ & $0.0 \%$ & $0.0 \%$ & $0.0 \%$ & $3.2 \%$ & $3.65 \%$ & $2.9 \%$ & $3.2 \%$ \\
\hline $70-80 \%$ & $0.0 \%$ & $0.0 \%$ & $0.0 \%$ & $0.0 \%$ & $0.3 \%$ & $0.39 \%$ & $0.29 \%$ & $0.33 \%$ \\
\hline 80-90\% & $0.0 \%$ & $0.0 \%$ & $0.0 \%$ & $0.0 \%$ & $0.03 \%$ & $0.04 \%$ & $0.03 \%$ & $0.03 \%$ \\
\hline $90-100 \%$ & $0.0 \%$ & $0.0 \%$ & $0.0 \%$ & $0.0 \%$ & $0.02 \%$ & $0.03 \%$ & $0.02 \%$ & $0.02 \%$ \\
\hline
\end{tabular}

Pairwise sequence percent identity of positive subsets (AS: All Sequences and GS: Gold Standard) for sequences with a length of $600 \mathrm{nt}$ and $20 \mathrm{nt}$ for donor and acceptor SS (values in bold correspond to the highest percentage of identity) 
[49]. Determining a pertinent sequence length is important because too short genomic regions would prevent the model from using important discriminatory sites, while too large genomic regions may introduce noise-inducing features and loss of accuracy [50]. We then built CNN prediction models for donor and acceptor SS, using these different sequence lengths. Figure 2 (and Additional file 2: Table S1 and S2) summarizes the prediction accuracies obtained by the models for each SS on the test sets.

We observe similar trends for the prediction of donor and acceptor SS with the AS and GS datasets. The average prediction accuracy increases for sequence lengths ranging from 20 to $200 \mathrm{nt}$ and then generally levels off, indicating that the model cannot find relevant genomic context features beyond this length. However, there are some differences between the datasets with different compositions (described in detail below). For example, for the AS_10 and GS_10 datasets (ratio 1:10 of positive to negative examples), the prediction accuracies are more homogeneous and higher than the other datasets. Interestingly, the sequence length has less effect for the AS_1 and GS_1 datasets, compared to AS_0 and GS_0 respectively. AS_1 (respectively GS_1) has the same balanced ratio of positive to negative examples as AS_0 (respectively GS_0), but the negative examples are more heterogeneous, consisting of exon, intron and FP sequences.

Based on this initial analysis, in the following experiments, we used a sequence length of $200 \mathrm{nt}$ for the prediction of donor and acceptor sites with the AS and GS dataset, to consider a genomic context that is neither too small nor too large.

\section{Impact of data quality}

As described above, the GS dataset contains only true SS from the 'Confirmed' gene sequences, while the AS dataset includes some noise (i.e. false SS) from 'Unconfirmed' sequences. To estimate the impact of this noise on model prediction, we compared the average accuracy of the AS models with the corresponding GS models for each SS (donor and acceptor) and for different dataset compositions, as shown in Fig. 3 (Additional file 1: Table S1).

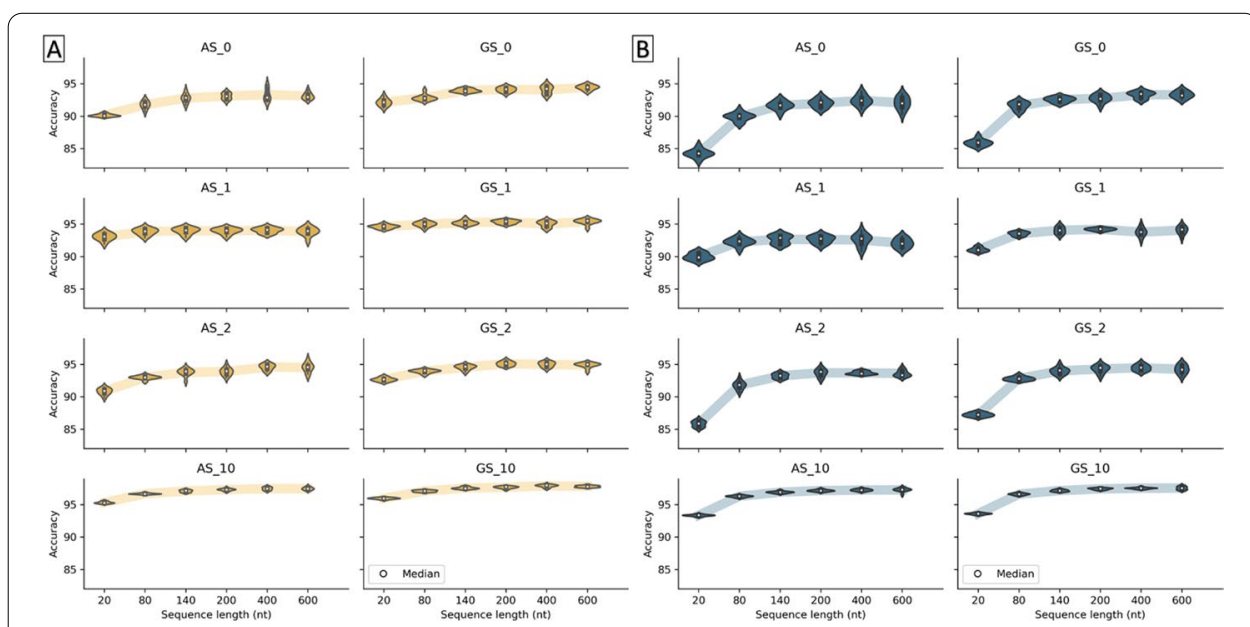

Fig. 2 Prediction accuracy according to input sequence length for each dataset (AS: All Sequences and GS: Gold Standard) for $\mathbf{A}$ donor and $\mathbf{B}$ acceptor SS 

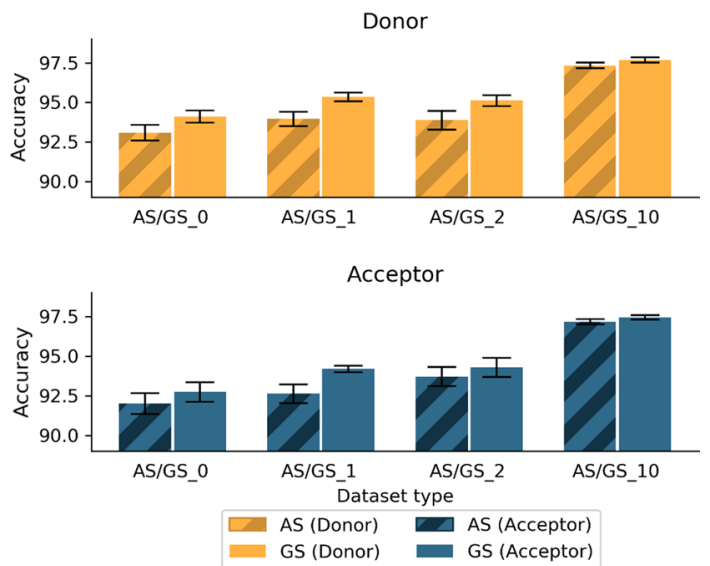

Fig. 3 Average prediction accuracy for donor and acceptor SS, using the AS and GS datasets (AS/ GS_0 = positive/negative ratio of 1:1 with only FP sequences in negative subset; AS/GS_1 = positive/negative ratio of 1:1 with exon, intron and FP sequences; AS/GS_2 = positive/negative ratio of 1:2 with only FP sequences in negative subset; AS/GS_10= positive/negative ratio of 1:10 with only FP sequences in negative subset). Standard deviations are indicated by black bars

As expected, the GS models achieved the best accuracy with an average of $94.11 \%$, 95.34\%, 95.11\% and 97.68\% respectively for the GS_0, GS_1, GS_2 and GS_10 donor datasets and $92.73 \%, 94.19 \%, 94.59 \%, 97.45 \%$ respectively for the acceptor datasets. In comparison, the AS models obtained an average accuracy of $93.09 \%(-1.02 \%), 93.95 \%$ $(-1.39 \%), 93.88 \%(-1.23 \%)$ and $97.33 \%(-0.35 \%)$ respectively for the AS_0, AS_1, AS_2 and AS_10 donor datasets, and $91.99 \%(-0.74 \%), 92.62 \%(-1.57 \%), 93.70 \%(-0.49 \%)$ and $97.17 \%(-0.28 \%)$ for the acceptor datasets. The results of unpaired t-tests (Additional file 1: Table S2) show that all differences between AS and GS datasets are statistically significant. Interestingly, the difference between AS_1 and GS_1 is the largest for both donor and acceptor models. Based on these results, we selected only the GS models for the following experiments.

\section{Impact of negative dataset composition}

While the definition of reliable positive examples is clearly essential, the construction of the negative dataset will also have an impact on the ability of CNN methods to distinguish between positive and negative examples. The prediction of SS is an intrinsically unbalanced problem, since in a protein coding gene the SS represent only a small proportion of the total nucleotide length. Therefore, to investigate the impact of the negative subset on prediction performance, we constructed a number of datasets with different types of negative sequences and different ratios of positive and negative examples. We designed two balanced datasets, both with a ratio 1:1 of positive to negative examples, but with either homogeneous (GS_0) or heterogeneous (GS_1) negative examples, as well as two unbalanced datasets with ratios of 1:2 (GS_2) and 1:10 (GS_10) of positive to negative examples. The unbalanced datasets both have heterogeneous negative examples. We then computed different metrics to evaluate the performance of each model on the test set, as shown in Fig. 4. 


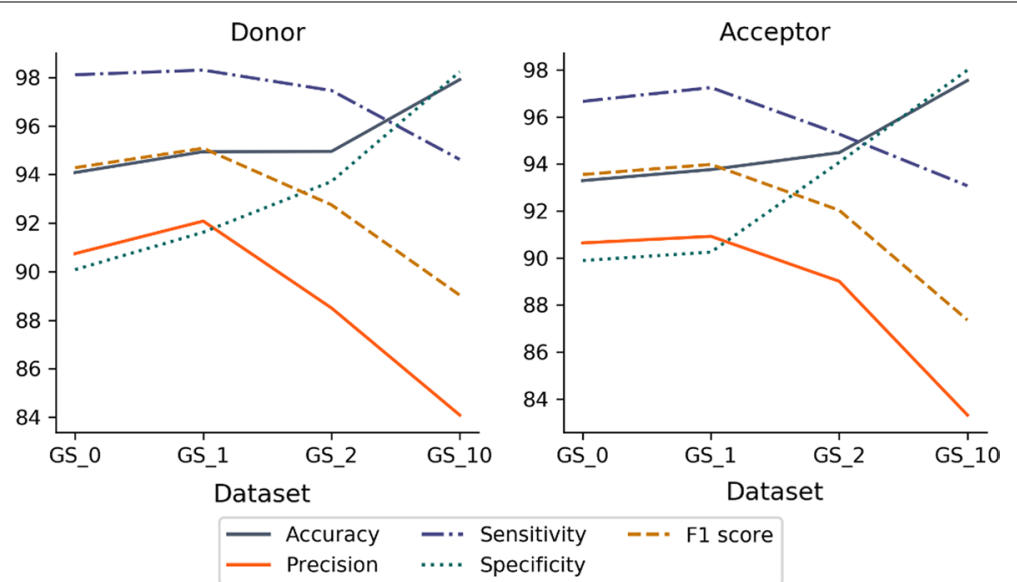

Fig. 4 Average values of the 5 performance metrics (accuracy, precision, sensitivity, specificity and F1 score) for each dataset composition and for each type of SS (donor or acceptor). GS_0 = positive/negative ratio of 1:1 with only FP sequences in negative subset, GS_1 = positive/negative ratio of 1:1 with exon, intron and FP sequences in negative subset, GS_2 = positive/negative ratio of 1:2 and GS_10 = positive/negative ratio of $1: 10$

The overall best performance for the test set is obtained using the GS_1 dataset, with a balanced number of positive and negative sequences and heterogeneous negative examples (exon, intron and FP sequences). Although the average accuracy is lower than for the unbalanced GS_10 dataset (positive/negative ratio of 1:10), the other metrics including the average F1 score and precision are higher for the GS_1 balanced dataset.

To confirm these results, the GS models were also evaluated on a set of 5 independent benchmarks (human, fish, fly, worm, plant) and using different metrics. The results are shown in Additional file 1: Figure S1A and B. The GS_1 dataset again obtains better overall performance metrics for both donor and acceptor SS, notably for fly, worm and plant species. Based on these results, we chose to consider only the GS_1 models in the following experiments.

\section{Performance of optimized CNN model}

Based on our initial analyses, we determined the optimal training set for the CNN models to predict donor and acceptor SS, namely the GS_1 dataset: a high quality balanced dataset with an equal number of positive and negative sequences, heterogeneous negative examples containing exon, intron and FP sequences and an input sequence length of $200 \mathrm{nt}$. For this optimized model, we further characterized the prediction performance of Spliceator averaged over a total of 10 experiments due to the random selection of negative sequences. The results are shown in Fig. 5 (Additional file 1: Table S3).

The average accuracy is $95.34 \%$ for the optimized donor model and $94.19 \%$ for the acceptor model. The precision of the models is similar, ranging from $92.50 \%$ (donor) to $91.73 \%$ (acceptor). We observed high sensitivity for both models, with $98.31 \%$ (donor) and $97.20 \%$ (acceptor), although the specificity is slightly lower, with $92.11 \%$ (donor) and 91.14\% (acceptor). Finally, the F1 scores are similar for both donor and acceptor with $95.32 \%$ for donor and $94.39 \%$ for acceptor SS. Thus, the average performance for the donor model is slightly higher than for the acceptor model, which 


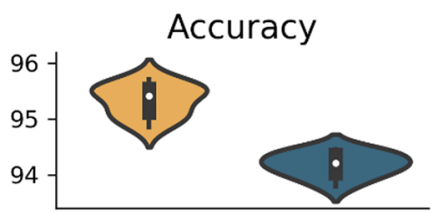

Sensitivity

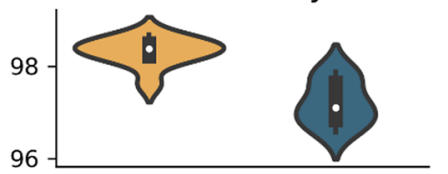

F1_score

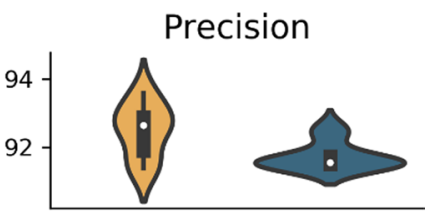

Specificity

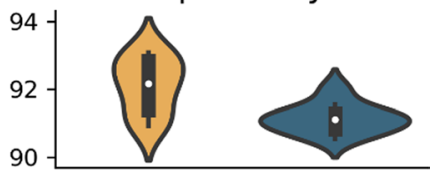

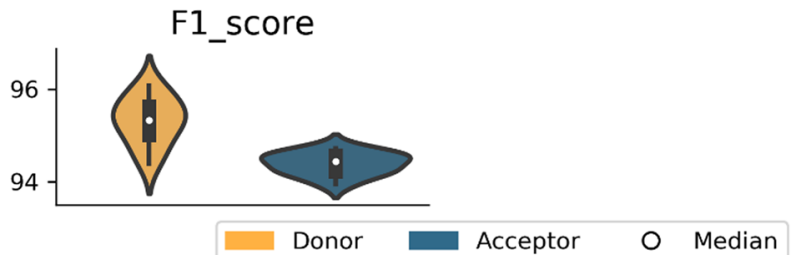

Fig. 5 Performance of optimized model (GS_1 dataset, positive/negative ratio of 1:1 with heterogeneous negative examples and input sequence length $=200 \mathrm{nt}$ ) averaged over 10 experiments

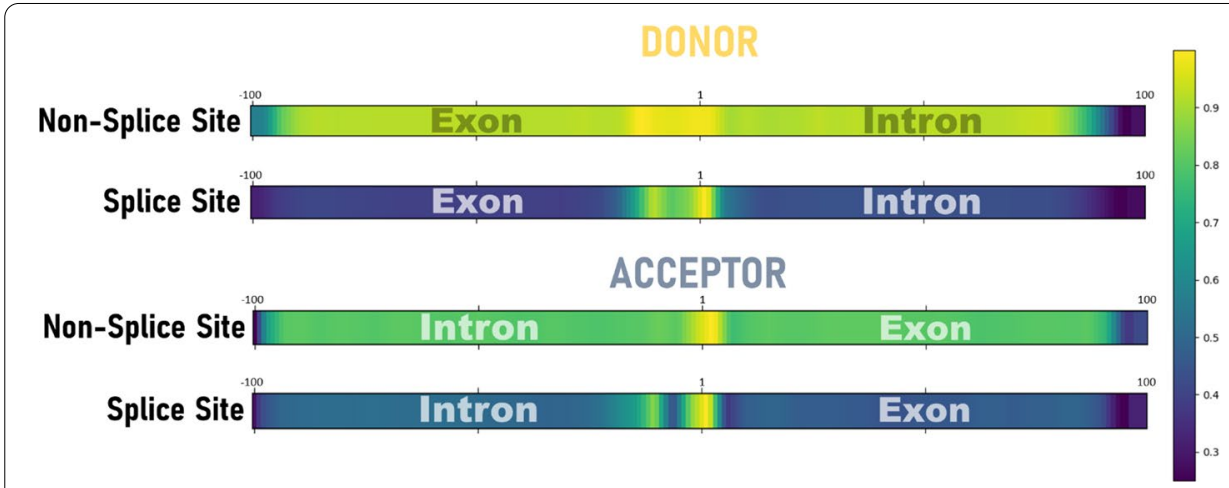

Fig. 6 Average heatmap of the two classes, non-Splice Site and Splice Site, for donor and acceptor SS, with colors ranging from yellow (very important nucleotide position) to dark blue (not important position). The dinucleotide characterizing the SS is located at positions 101-102 for the donor and acceptor SS

might be explained by the fact that the donor SS consensus motif is more conserved than the acceptor SS motif (Table 1). The acceptor SS also contains a low complexity PPT sequence, which may complicate predictions.

\section{Model interpretability}

In this section, we focus on the nucleotide regions that influenced the models during the learning step. Using the Grad-CAM method (see "Methods" section), we measured the impact of each nucleotide position in the input sequences, thus allowing us to highlight the most important regions of these sequences that are determining factors in the learning step of the model. We calculated 10,000 heatmaps per class and the average heatmap for each SS model (donor and acceptor) and each class (non-SS, 
SS) is shown in Fig. 6. Therefore, this representation shows only the most important features that the models use.

The heatmaps show that in order to classify sequences as non-SS, both models are based on elements of the whole sequence (score $>0.8$ ) with the exception of the 5 ' and 3 ' extremities (score $<0.4$, probably due to the $\mathrm{CNN}$ processing), although we observe a higher score $(>0.9)$ close to the positions $1-2$. The heatmaps for sequences containing donor or acceptor SS are more specific than for the non-SS sequences. For the donor sequences, the region around the SS ( $10 \mathrm{nt}$ upstream and downstream) seems to be more influential, with a predominance for the upstream exonic side. The positions 1 and 2, representing the GT dinucleotide, have the highest scores as expected. For the acceptor sequences, the central region around the AG dinucleotide is also the most important, although it is less well delineated than for donor SS. We observe an upstream intronic region of about $10 \mathrm{nt}$ that seems to slightly impact learning (score $>0.6$ ), which probably corresponds to the PPT. A second upstream region from position -50 is also influential, possibly covering the BPS known to be generally located around $40 \mathrm{nt}$ upstream of the acceptor site [51], although some BPS may be more distant up to a distance of $400 \mathrm{nt}$ [52, 53]. Interestingly, the downstream exonic region also seems to play a role in the training process for the acceptor SS (score $>0.5$ ).

\section{Comparison with existing SS prediction methods}

In order to compare the performance of the Spliceator models with other state-ofthe-art methods, namely NNSplice, MaxEntScan, DSSP and SpliceFinder, we used six independent benchmarks from a wide range of organisms (see "Methods" section). The performance metrics are shown in Table 2.

For the donor SS prediction, Spliceator obtains the best average accuracy of $92.82 \%$, with an average increase of $+25.9,+2.6,+2.04$ and $+1.15 \%$ compared to NNSplice, SpliceFinder, DSSP and MaxEntScan respectively. For the acceptor SS prediction, Spliceator obtains the second best average accuracy, with $89.02 \%(-1.26 \%)$ compared to SpliceFinder with $90.28 \%$, although Spliceator is more accurate on the Worm and Plant benchmarks. To further investigate the reasons for the different performances, we considered four other metrics, including the precision, sensitivity, specificity and F1 score. A high precision indicates that the program predicts few FP. Spliceator obtains the second best average precision of $89.88 \%$ for the donor SS and $86.23 \%$ for the acceptor SS, behind DSSP (96.17\% for donor and $92.28 \%$ for acceptor SS). SpliceFinder, which has generally good accuracy, obtains lower precision $(74.92 \%$ for the SS donor and $69.52 \%$ for the SS acceptor). Sensitivity and specificity are two inseparable metrics. They describe the proportion of well predicted elements and their quality, i.e. if elements have been correctly predicted.. The F1 score combines the precision and the sensitivity metrics and provides a more global view of the number of correctly predicted elements. Spliceator obtains the best average F1 score for both donor and acceptor SS, with 93.08\% (+1.15\%) and 89.4\% $(+3.83 \%)$ compared to the second best program for donor SS, MaxEntScan with an F1 score of $91.93 \%$ or acceptor SS, DSSP with a F1 score of $85.57 \%$. Figure 7 shows the accuracy and F1 score for each program on the individual benchmarks containing SS from different organisms. While most of the programs tested achieve high scores on the vertebrate sequences (human and fish), reflecting their training sets focused on human SS, 


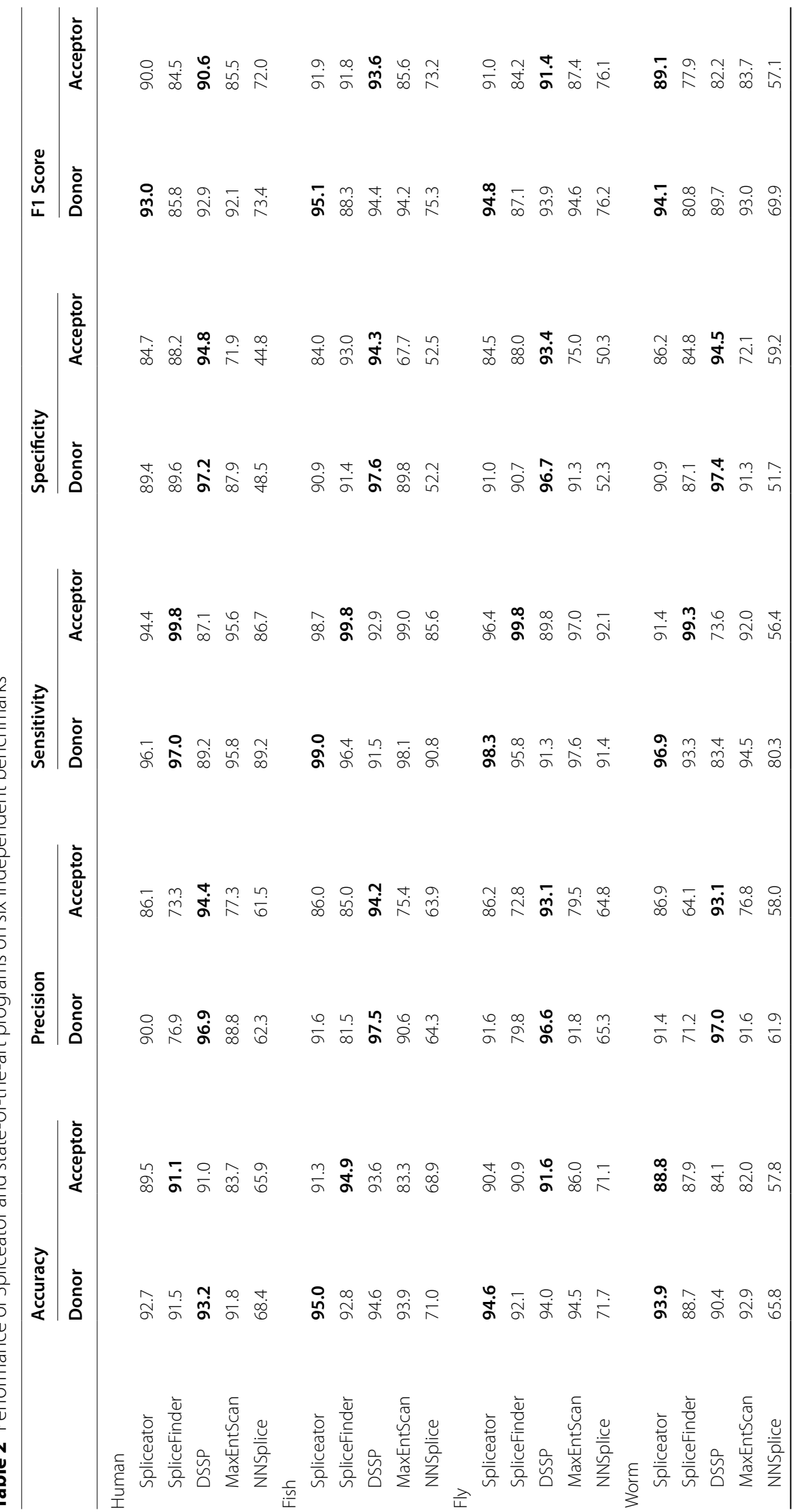




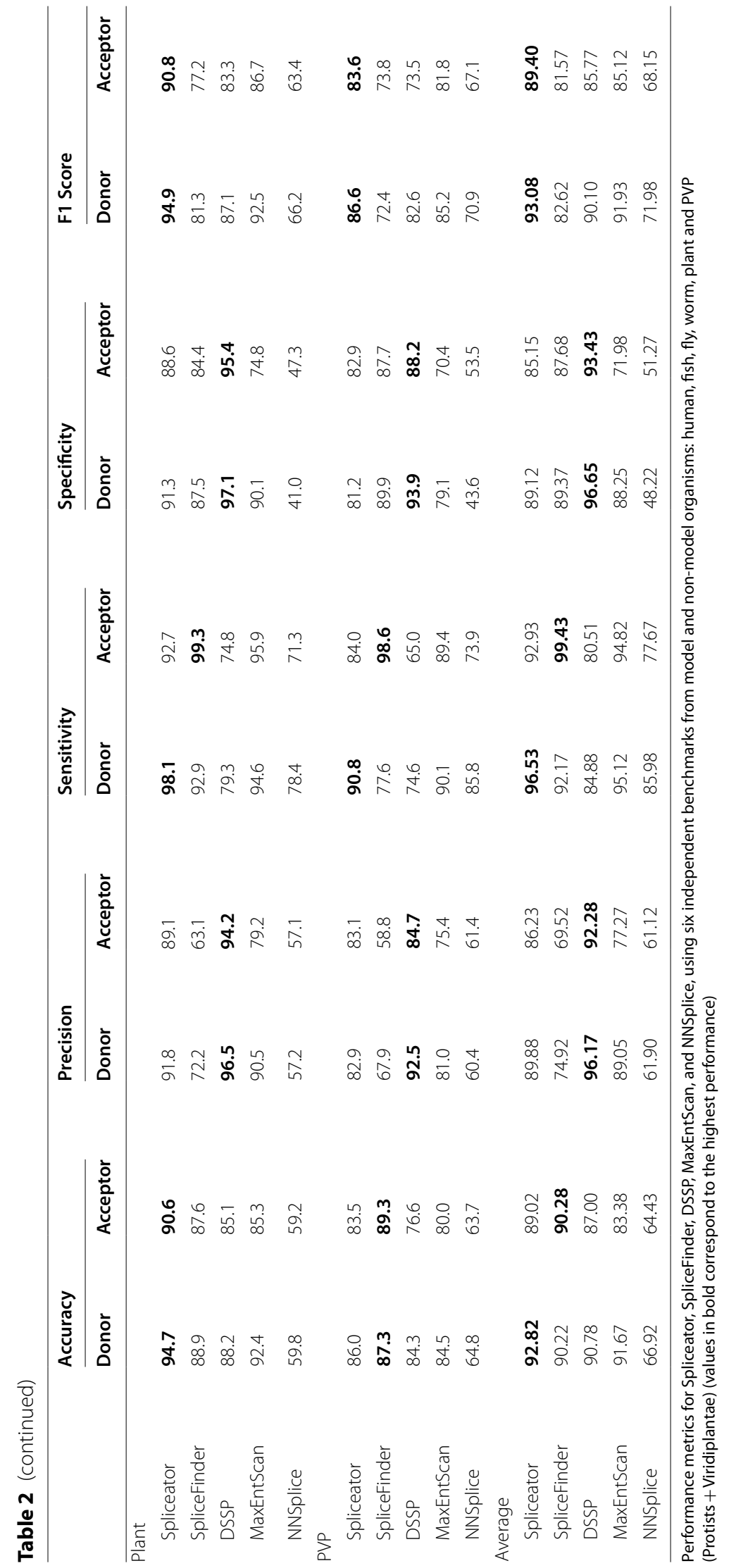




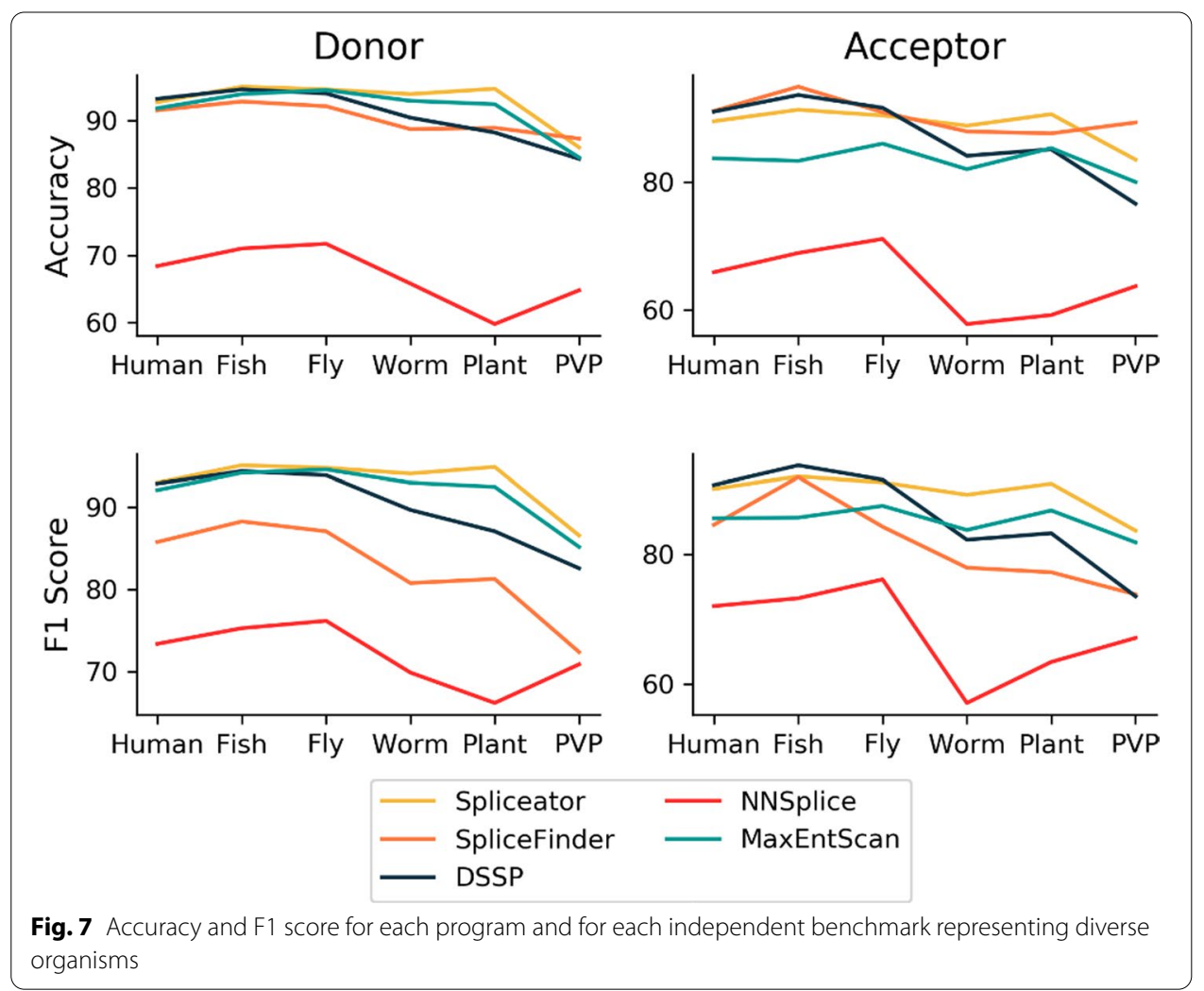

Spliceator performance is generally better on the more distant organisms (worm, plant and PVP).

Finally, for each model, we observe that the predictions of the donor SS are slightly better than those of the acceptor SS. This difference is probably due to the divergence of the acceptor SS motif, but also to the genomic context around it which seems to be more complex.

\section{Discussion}

Thanks to high-throughput technologies, as well as the development of computing power, huge amounts of data can now be exploited by DL algorithms and produce remarkable results [54-57]. In particular, CNN are increasingly used in the field of bioinformatics [58-60], for example to detect specific patterns in a genomic sequence [61] where the reduced number of parameters allows for better generalization compared to other ML methods. Moreover, maxpooling techniques allows the algorithm to focus on the local features that it considers most important.

In this context, we have developed a SS prediction program called Spliceator, based on a three-layer convolutional CNN. Despite the recent use of RNA-seq to accurately identify SS, it is currently impossible to obtain experimental data for the full panel of transcripts for all tissues and all developmental stages. Thus, ab initio SS prediction programs that rely only on the genomic sequence remain essential. Clearly, it would be ideal to couple ab initio prediction programs such as Spliceator with RNA-seq based programs in genome annotation tools or workflows. 
The accuracy of ab initio algorithms is dependent on the quality of the data used during the training step. Indeed, although neural networks are getting deeper and more complex [62, 63], the fact remains that data is the cornerstone of artificial intelligence. Consequently, the majority of SS prediction programs use sequence data from humans (such as GRCh38 or HS3D [64]) or other model organisms [36, 39, 65], where high quality, expert-refined data is available. For non-model organisms, it is more difficult to find accurate training data and very few $\mathrm{CNN}$ methods have been designed specifically to predict SS in non-model organisms. Since SS and other regulatory motifs may be conserved across similar species [20], some work has been done to try to transfer models trained on model organisms to related organisms, for example between different vertebrate genomes [66]. Others have built cross-species models for specific clades, such as animals or plants using Helixer [67], but unfortunately the source code for this program is not yet stable (according to the authors). The aim of our work was to extend the idea of cross-species models to conceive universal SS prediction models (one for each SS), that are applicable to a wider range of organisms.

For the training of the Spliceator models, we focused on the construction of a multispecies dataset that is as representative as possible of the eukaryotic domain (from primates to protists). This dataset is based on an extension (G3PO+) of the gene prediction benchmark G3PO. Since high-quality, genome-wide annotations are not available for the 147 species in this dataset, we developed a protocol based on expert-guided comparative sequence analysis in order to identify reliable SS in a subset of genes. Since the G3PO+ gene sequences are evolutionarily related, we eliminated redundant sequences, which could cause potential bias of sequence over-representation and thus a risk of overfitting. We also made an effort to respect the proportions of non-canonical SS (2.2\% donor and $1.3 \%$ acceptor) found in real-world data [21].

To investigate the impact of the quality of the initial training data on the CNN models, we extracted data from public databases such as Ensembl [4] and UniProt [68], where it has been estimated that many proteins (with the exception of Swiss-Prot, which represents $0.3 \%$ of UniProt) have errors [69]. We then built a dataset called 'All Sequences' (AS), that includes some badly predicted gene sequences [45] and thus introduces noise in the form of wrong or missing SS. We compared the CNN model trained on the AS dataset with a second model trained on a 'Gold Standard' (GS) dataset, which was cleaned by removing all error-prone sequences. Since our results showed that the quality of the data had a significant impact on the accuracy of the models, we conclude that quality control and data cleaning steps are essential in order to obtain better results.

We also tested the impact of other parameters, such as the length of the input sequences. It is important to carefully select the size of the genomic sequence in order to take into account different important elements such as regulatory elements (ESE, ESS, ISE or ISS [70]), the BPS [71] and the PPT [22] that can be kept and help the algorithm to generalize. All these elements constitute intrinsic signals that are indispensable for the spliceosome to accurately recognize the SS. In order to include enough cis elements without introducing too much noise, we chose an input sequence length of $200 \mathrm{nt}$ for both donor and acceptor models. Unfortunately, many other external signals impacting SS recognition by the spliceosome cannot be detected by current methods such as the secondary structure of RNA [72], or the transcription speed of polymerase II [73]. 
Finally, we investigated the impact of the negative examples and the use of balanced or unbalanced datasets, in terms of the ratio of positive to negative examples. SS prediction is an inherently unbalanced problem, because the number of nucleotides involved in a SS is much smaller than the number of non-SS nucleotides. The results confirmed the hypothesis that an unbalanced dataset was more prone to overfitting because one of the classes is overrepresented [66]. Moreover, the heterogeneous negative examples provided better performance. All these tests allowed us to optimize our method and improve prediction performance.

In order to estimate the performance of Spliceator on independent genome data, we used six different benchmarks from diverse organisms and compared Spliceator with a number of state-of-the art programs, including two other CNN-based methods. As expected, the more recent $\mathrm{CNN}$-based methods generally achieved higher performance metrics than the older prediction methods that used either neural networks or maximum entropy distributions approaches. We calculated a number of different performance metrics, since the most suitable metric to measure 'good' performance will depend on the specific user application. For example, accuracy is useful when the true positives and true negatives are more important, while the F1 score is used when the false positives and false negatives are crucial. Spliceator achieved the highest accuracy (92.82\%) for the donor SS, and the second best accuracy (89\%) for the acceptor SS. In terms of F1 score, Spliceator outperformed the current state-of-the art programs for both donor (93.08\%) and acceptor (89.40\%) SS. Interestingly, Spliceator performed very well on the human benchmark even though it was trained with only 45 human genes. However, a major strength of Spliceator is that it maintains good performance over a wide range of organisms, from human to protists. Our results thus showed that a universal SS prediction program is feasible, and hopefully performance can be further increased in the future by including more divergent species data in the model.

\section{Conclusions}

Here, we present a new approach to train Spliceator, a universal splice site prediction program based on a high-quality dataset from diverse eukaryotic organisms (from primates to protists). We highlighted the inherent link between data quality and the performance of prediction programs based on machine learning algorithms. We also showed that including high quality multi-species data can result in accuracy equivalent to other state-of-the-art SS prediction programs. In the future, it would be interesting to include more data from other species, but also to test other types of network architecture in order to extract new high-level features. Moreover, as some of the extracted features are highly conserved, it would be interesting to use our model to perform transfer learning for gene annotation of other organisms.

\section{Methods}

\section{Data collection}

Initial datasets were constructed for each type of SS, in order to establish two separate models: one to predict donor SS and one to predict acceptor SS. The models developed in this study are based on supervised learning allowing the classification of entries in two classes (0: nucleotide not involved in SS, and 1: nucleotide involved in SS). Thus, 
each dataset is constructed from a positive subset containing SS sequences and a negative subset containing non-SS sequences.

To build the positive and negative subsets, gene sequences from the multi-species benchmark G3PO [45] were used. G3PO is based on 147 phylogenetically disperse organisms and contains 1793 sequences including 20 human Bardet-Biedl Syndrome (BBS) genes (Additional file 1: Table S4) and their orthologous sequences (ranging from primates to protists) extracted from the OrthoInspector database v3.0 [74]. Following the same methodology implemented in G3PO, we extended the original dataset by adding 948 sequences from 25 human genes responsible for myopathies and their orthologs from 47 metazoans (Additional file 1: Figure S2). The 948 protein sequences in the extended dataset (called G3PO+) were analyzed according to the G3PO protocol in order to classify them into two categories: those without gene prediction errors (called 'Confirmed') and those that contain at least one error (called 'Unconfirmed'). Errors include insertions, deletions and mismatches in the $\mathrm{N}$-terminal, C-terminal or internal regions. This protocol allows to verify the quality of the data and ensures that the SS present in the 'Confirmed' sequences are biologically true, i.e. they are recognized by the spliceosome. Table 3 summarizes the composition of the G3PO+ dataset.

\section{Construction of a series of training and test sets}

Based on the gene sequences in the G3PO+ benchmark, we constructed a series of datasets used to train the Spliceator models and estimate the effect of various parameters on their prediction performance. Figure 8 shows an overview of the dataset construction process. The positive and negative subsets are described in the following sections.

\section{Positive subsets}

The 2741 G3PO+ sequences are classified into 2 categories, either 'Confirmed' (1361 sequences) because they were annotated 'error-free', or 'Unconfirmed' (1380 sequences) because they contained at least one gene prediction error. For each $\mathrm{G} 3 \mathrm{PO}+$ sequence, genomic sequences and exon maps were retrieved from the Ensembl database [4] release 87, and the SS were extracted, flanked by a $\pm 300 \mathrm{nt}$ environment. A verification was made to ensure that no sequences containing undetermined nucleotides (noted 'N') were selected. The GS datasets contain only SS from the 1361 'Confirmed' sequences. Thus, the same positive subset for each GS dataset (GS_0, GS_1, GS_2 and GS_10) contains 10,973 donor and 11,179 acceptor SS sequences, where each sequence is of length $600 \mathrm{nt}$ with the GT (donor) or AG (acceptor) dinucleotide in the central position (301 and 302). In contrast, the

Table 3 Composition of the original G3PO and extended G3PO+ datasets

\begin{tabular}{lccc}
\hline & G3PO & Extension & G3PO+ \\
\hline Confirmed & 889 & 472 & 1361 \\
Unconfirmed & 904 & 476 & 1380 \\
Total & 1793 & 948 & 2741 \\
\hline
\end{tabular}

To build the G3PO+ dataset, we retrieved orthologous sequences for 45 human genes and performed multiple sequence alignments. Each sequence was then checked to identify those that contained no errors, called 'Confirmed', and those that contained at least one error, called 'Unconfirmed' 


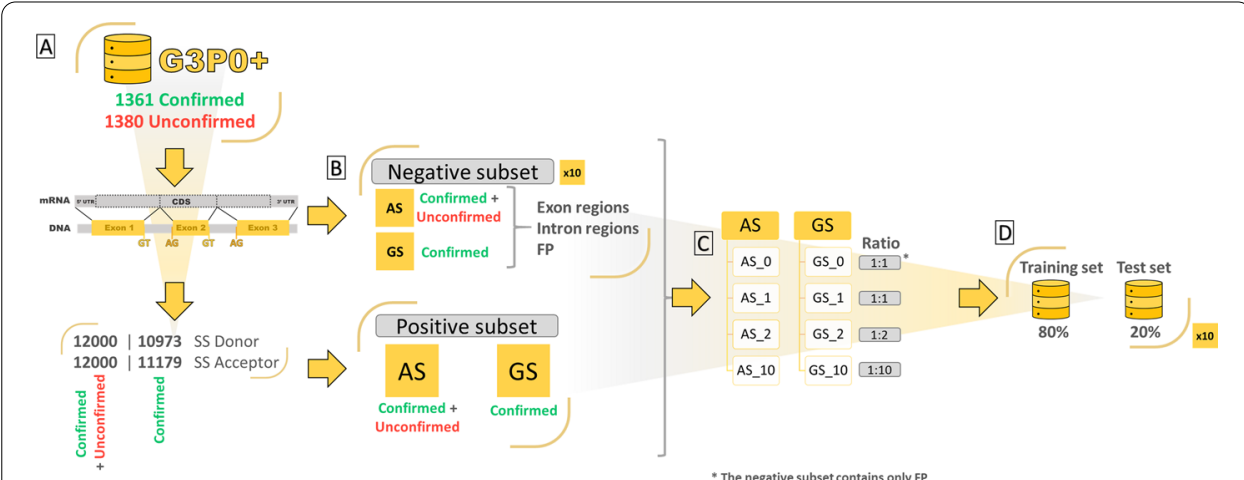

Fig. 8 Overview of the construction of the training and test sets. A DNA sequences and exon maps are recovered for each G3PO+ gene. B The AS (All Sequences) positive subset includes the SS of all G3PO+ 'Confirmed' and 'Unconfirmed' sequences. The GS (Gold Standard) positive subset includes only the SS of the 'Confirmed'sequences. Ten negative AS subsets and ten negative GS subsets are then constructed by random sampling of the exon, intron and FP regions of the corresponding genomic sequences. C Four AS and four GS datasets are then constructed with different ratios of positive and negative SS (described in Table 4). D Finally, the training and test sets are formed by shuffling the positive and negative sequences (10 times for each AS and GS dataset)

AS datasets contain SS from all $2741 \mathrm{G} 3 \mathrm{PO}+$ sequences, including both 'Confirmed' and 'Unconfirmed' sequences. Thus, the AS datasets are representative of the data present in the public databases, as no pre-processing has been performed on the data, and therefore they include a certain number of errors. To eliminate any bias from the size of the datasets, an equivalent number of SS sequences were used (12,000 donor and 12,000 acceptor) to form the same positive subset for each AS dataset (AS_0, AS_1, AS_2 and AS_10).

In order to test the impact of the genomic context, each dataset is provided in six different versions, according to the defined length of the input sequences. Sequence lengths selected for this study are 20, 80, 140, 200, 400 and $600 \mathrm{nt}$, where the SS is always in the central position. To reduce redundancy, for each sequence length, duplicate sequences are removed. As the length of the sequences decreases, the number of duplicates increases, reducing the size of the data sets (especially for 20 nt sequences). Figure 9 (Additional file 1: Table S5) summarizes the composition of the AS and GS positive subsets according to sequence length. Each SS is described according to its type, either canonical (i.e. GT for donor site and AG for acceptor site) or non-canonical. The number of non-canonical donor and acceptor SS present in each AS and GS dataset for each sequence length is also shown in Fig. 9 (Additional file 1: Table S5). In addition, Fig. 10 shows the sequence logos of the canonical and non-canonical donor and acceptor SS motifs from the AS and GS dataset sequences. The sequence logos were made with the program WebLogo v3.7.4 [75].

\section{Negative subsets}

Two negative subsets were first constructed. The first one is composed only of FP sequences, i.e. randomly selected regions in the G3PO+ sequences in both 'Confirmed' and 'Unconfirmed' sequences (for AS dataset) or only 'Confirmed' sequences (for GS dataset), with a GT or AG dinucleotide (depending on the type of SS), in the central 


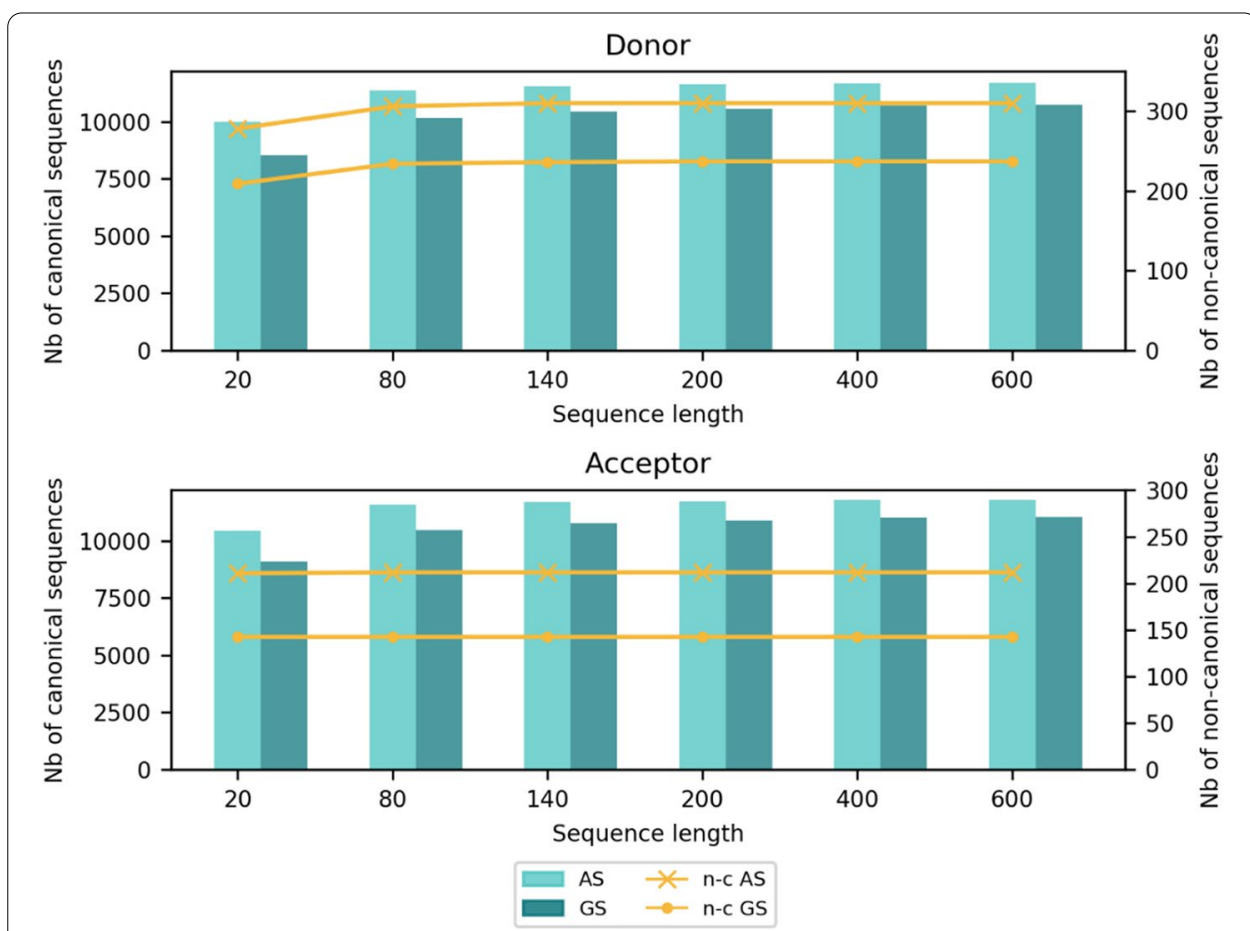

Fig. 9 Number of canonical (bar) and non-canonical ( $n-c)$ (line) sequences for each positive subset (AS and GS) and for each sequence length

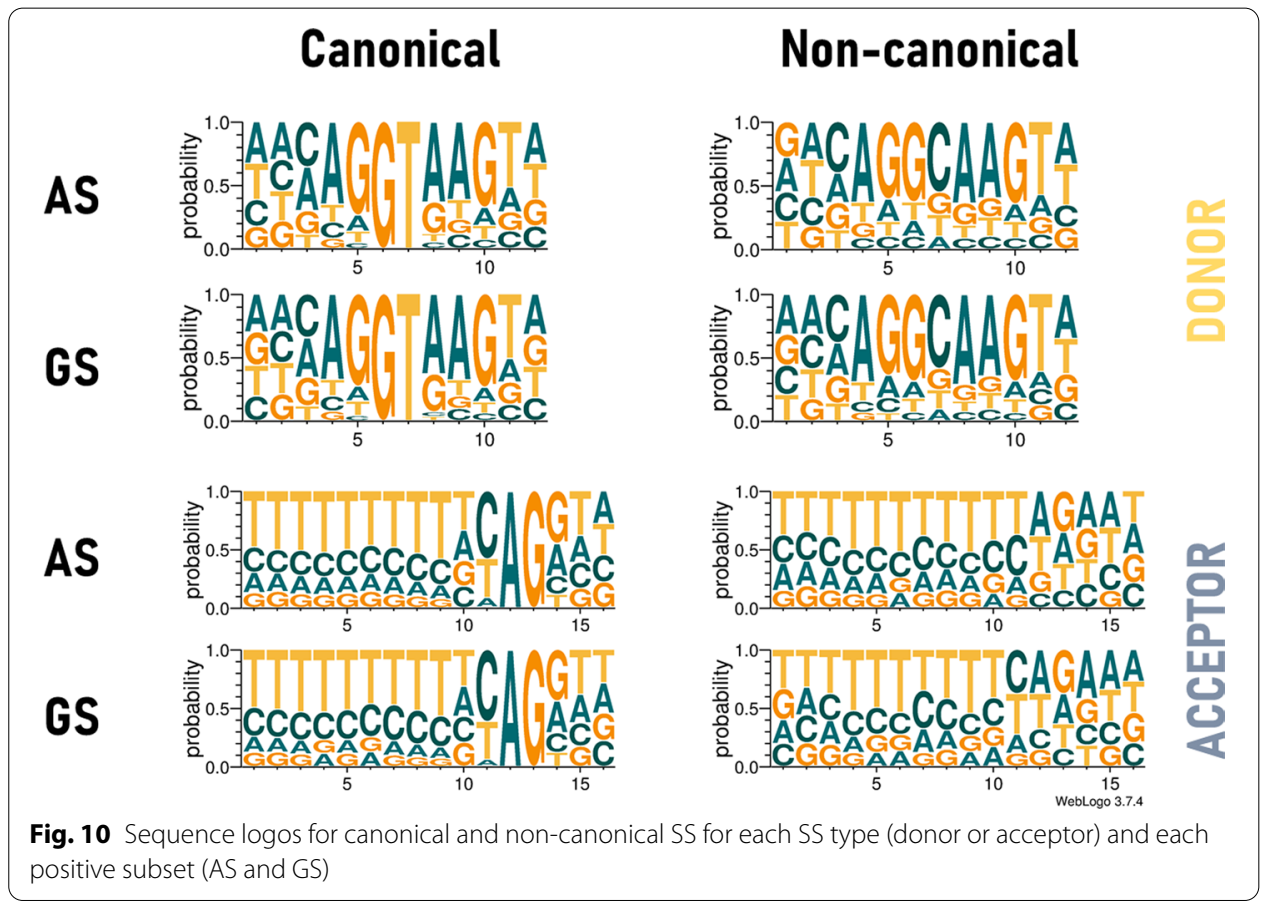

position (e.g. 301-302 for length $=600 \mathrm{nt}$ ) that do not correspond to a SS identified in the positive subsets. The second type of negative subset is composed of 3 categories of sequences extracted from the G3PO+ dataset: 
- Exon sequences: randomly selected exon regions,

- Intron sequences: randomly selected intron regions,

- False positive SS: randomly selected GT or AG dinucleotides.

Negative subsets were also constructed with different numbers of sequences depending on the size of the positive subset: (i) with a ratio 1:1 we have the same number of positive and negative sequences, (ii) with a ratio 1:2 we have twice as many negative sequences as positive sequences and (iii) with a ratio 1:10 we have ten times more negative sequences than positive sequences. As for the positive subsets, identical redundant sequences and sequences containing undetermined ' $N$ ' characters were removed. Finally, as the selection of the negative sequences is random, 10 random selections were made, in order to obtain 10 different negative subsets and to eliminate potential random bias due to a specific data sampling.

\section{Data composition strategies}

By combining the same positive subset with different negative subsets, a number of datasets were constructed in order to measure the impact of different parameters, including the type of negative examples used (heterogeneous $=$ exons, introns and FP or homogeneous=only FP), the use of balanced or unbalanced datasets defined by the ratio of positive to negative examples, and data quality (AS vs. GS). In total, eight datasets were established, summarized in Table 4.

\section{Sequence identity}

A sequence similarity search was performed on the whole sequences with a length of $600 \mathrm{nt}$ and $20 \mathrm{nt}$ from the AS and GS positive subsets. Each sequence was compared to all the others and the pairwise percent identity was defined by:

Table 4 Composition of the 8 datasets

\begin{tabular}{|c|c|c|c|c|c|c|c|}
\hline \multirow[t]{2}{*}{ Dataset } & \multirow[t]{2}{*}{ Quality of sequences } & \multicolumn{3}{|c|}{ No. of positive sequences } & \multirow{2}{*}{$\begin{array}{l}\text { No. of } \\
\text { negative } \\
\text { sequences }\end{array}$} & \multirow{2}{*}{$\begin{array}{l}\text { Type of negative } \\
\text { sequences }\end{array}$} & \multirow[t]{2}{*}{ Ratio } \\
\hline & & Donor & & Acceptor & & & \\
\hline AS_0 & \multirow{4}{*}{$\begin{array}{l}\text { Unconfirmed and } \\
\text { confirmed }\end{array}$} & \multirow[t]{4}{*}{12,000} & \multirow{4}{*}{\multicolumn{2}{|c|}{12,000}} & 12,000 & FP only & \multirow[t]{2}{*}{$1: 1$} \\
\hline AS_1 & & & & & 12,000 & $\begin{array}{l}4000 \text { exons, } 4000 \\
\text { introns and } 4000 \mathrm{FP}\end{array}$ & \\
\hline AS_2 & & & & & 24,000 & FP only & $1: 2$ \\
\hline AS_10 & & & & & 120,000 & FP only & $1: 10$ \\
\hline GS_0 & \multirow[t]{4}{*}{ Confirmed } & \multirow[t]{4}{*}{10,973} & \multirow{4}{*}{\multicolumn{2}{|c|}{11,179}} & 11,000 & FP only & \multirow[t]{2}{*}{$1: 1$} \\
\hline GS_1 & & & & & 11,000 & $\begin{array}{l}3650 \text { exons, } 3650 \\
\text { introns and } 3700 \mathrm{FP}\end{array}$ & \\
\hline GS_2 & & & & & 22,000 & FP only & $1: 2$ \\
\hline GS_10 & & & & & 110,000 & FP only & $1: 10$ \\
\hline
\end{tabular}

Composition of the 8 datasets used to study the impact of (i) the type of negative examples (only FP sequences vs. heterogeneous data with exons, introns and FP sequences), (ii) the ratio of positive to negative examples (1:1, 1:2 and 1:10), (iii) data quality ('Confirmed' and 'Unconfirmed' sequences in the AS datasets vs. only Confirmed sequences in the GS datasets

FP, False Positive; GS, Gold Standard; AS, All Sequences 


$$
\% \text { Identity }=\left(\frac{\text { Number of identical nucleotide }}{\text { Length of sequence }}\right) * 100
$$

\section{Preparation of datasets for CNN}

\section{Training and test sets}

For each dataset described above, a random selection was performed to form a training set containing $80 \%$ of the sequences and a test set containing $20 \%$ of the sequences. Since there are 10 different negative datasets, there are 10 different training and test sets. Figure 11 (Additional file 1: Table S6 A and B) summarizes the average number of
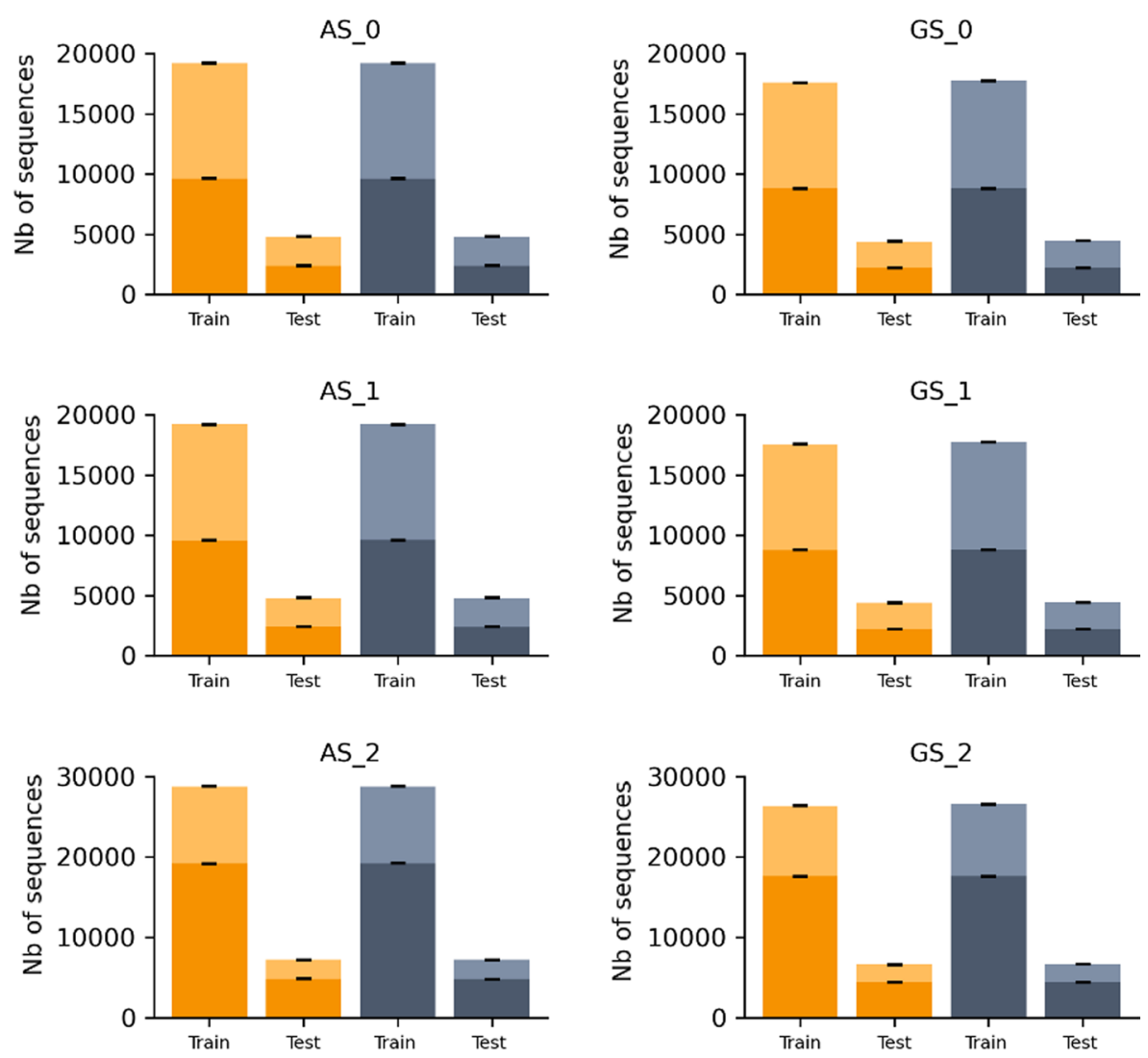

AS_10

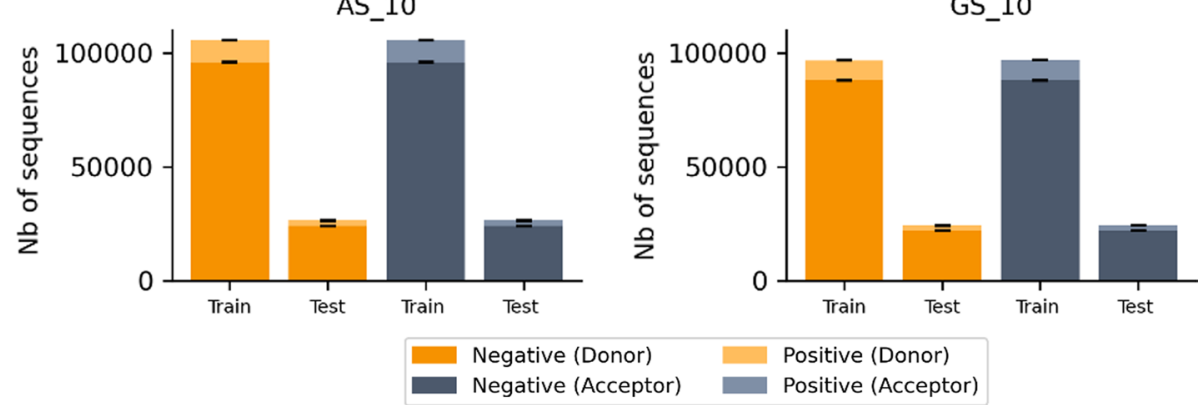

Fig. 11 Average number of positive and negative sequences in training and test sets, for all AS and GS datasets, according to SS type. Standard deviations are indicated by black bars 
negative and positive sequences in all training and test sets for each dataset (AS and GS) and for donor and acceptor SS.

\section{Data encoding}

For efficient exploitation of the genomic sequences in the training and test sets, a onehot encoding step was performed. Each nucleotide of an input sequence was converted into a binary vector of size 4 . Adenine is encoded by the vector $(1,0,0,0)$, Cytosine by $(0,1,0,0)$, Guanine by $(0,0,1,0)$ and finally Thymine is encoded by $(0,0,0,1)$. In the case where an external sequence contains indeterminate ' $\mathrm{N}$ ' nucleotides, $e . g$. when users test their own sequences, the vector $(0,0,0,0)$ is used. Thus, each output sequence is a first order tensor (vector) of size $\mathrm{W}$, where $\mathrm{W}$ is the length of the input sequence, with 4 channels representing the one-encoding. Finally, the shape of the input is: $\mathrm{S} \times \mathrm{H} \times \mathrm{W} \times \mathrm{C}$, where $S$ is the number of input sequences, $H$ is the height of the $1 D$ vector (so $H=1$ ), W is the width of the vector corresponding to the length of the input sequences and $\mathrm{C}$ is the number of channels from one-hot encoding. Figure 12 summarizes the data encoding.

Finally, the shape of the input is $\mathrm{S} \times \mathrm{H} \times \mathrm{W} \times \mathrm{C}$ ( $\mathrm{S}=$ number of input sequences, $\mathrm{H}=$ height of the vector (here equal to 1 because the vector is 1 dimensional), $W$ is the length of each input sequence and $\mathrm{C}$ is the number of channels).

\section{Convolutional Neural Network}

Models were constructed and trained for each type of SS (donor or acceptor) independently. The models result from supervised learning, where genomic sequences (input) are coupled with class labels 0: non-SS and 1: SS. The CNN then applies filters on each input sequence and tries to modify the weights of these filters to improve the predictions thanks to the back-propagation algorithm. The filters allow to extract pertinent features/ patterns within the input data. The output of the $\mathrm{CNN}$ is a vector of size 2, corresponding to the non-SS (0)/SS classes (1). The implementation of the CNN, as well as the training of the models, was done in Python v3.7, with Tensorflow v2.4.1 [76], the API Keras v2.3.1 and the Scikit-learn library v0.23.2 [77].

\section{Architecture}

We constructed a CNN architecture for donor or acceptor prediction, composed of a series of three convolutional layers over a single spatial dimension. The layers are

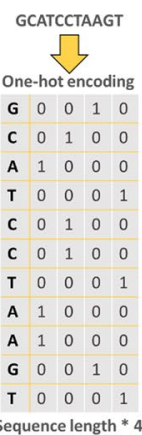

Fig. 12 Data pre-processing. Input sequences are converted in one-hot encoding. The result is a 1D vector of size $W$, where $W$ is the length of the input sequences, with 4 channels 
composed of 16, 32 and 64 filters of sizes 7, 6 and 6 respectively, with a stride of 1 . Between each convolution layer, a maxpooling layer of size $2 \times 1$ with a stride of 2 is added. Maxpooling allows to under-sample the data by reducing their size, while preserving the features that seem important. A dropout layer [78] is also added between each convolution layer to inactivate $20 \%$ of neurons. Then, a data flattening step (flatten layer) is performed in order to generate a vector exploitable by the fully connected layer containing 100 neurons. The last layer is the final output layer, containing two neurons that return the results of the classification. The neurons of each layer are activated by a Rectified Linear Unit (ReLU) activation function, except for the last layer where the activation function is Softmax in order to establish probabilities for each neuron and thus to predict a class according to the highest probability. Figure 13 summarizes the $\mathrm{CNN}$ architecture.

\section{Training process}

During the training process, the training set is divided into two parts, to generate an evaluation set (containing 15\% of the sequences) that allows to control the learning of the network and avoid overfitting. The cross-entropy function is used as a loss function and the Adamax optimization algorithm [79] is applied with a learning-rate of $1 \mathrm{e}^{-5}$. Finally, the training is performed during 400 epochs with a batch-size of 32 .

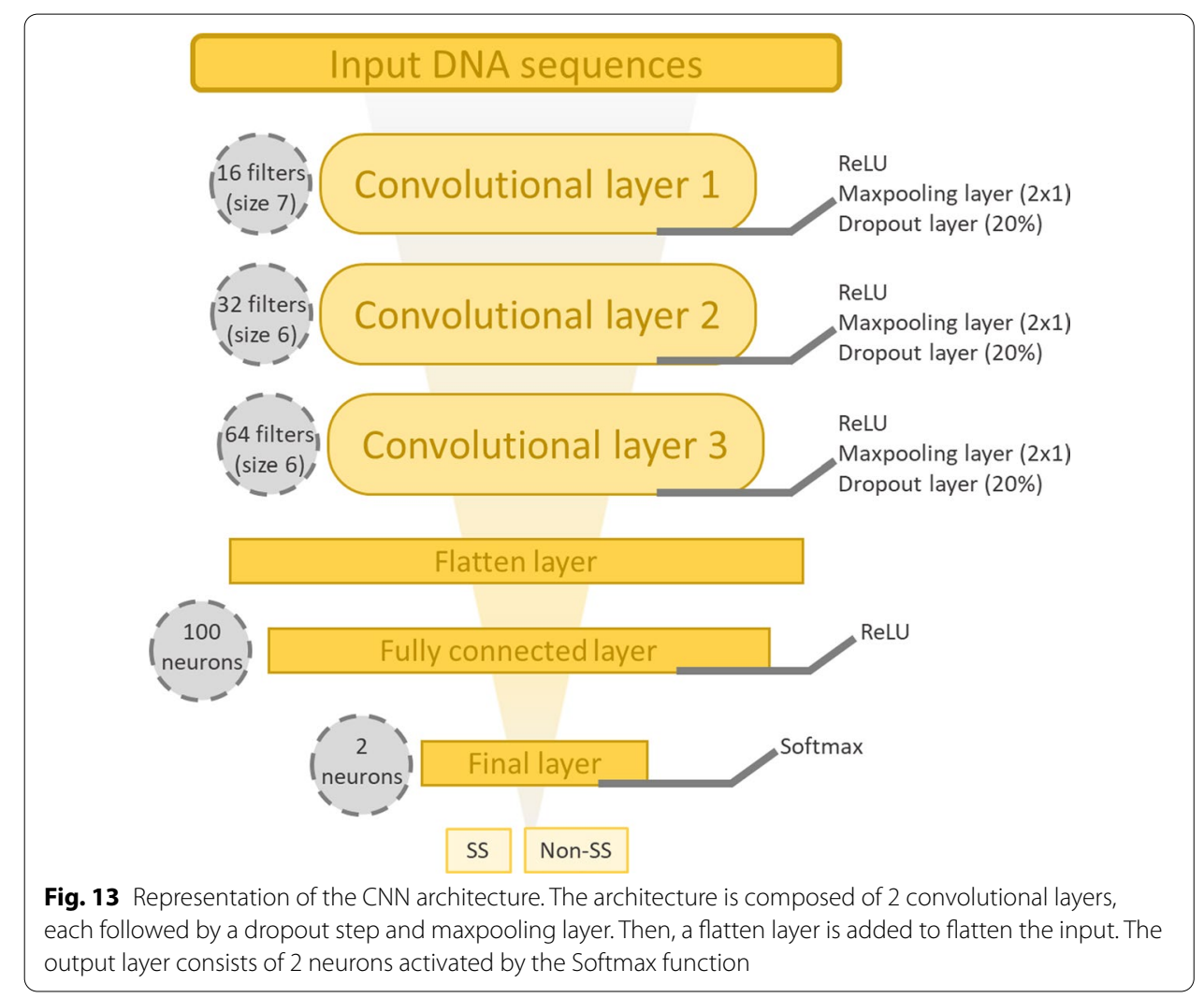




\section{Evaluation}

\section{Metrics}

SS are considered as true positives (TP) if they are correctly predicted and false positives (FP) otherwise. Nucleotides that do not correspond to a SS are considered as True Negatives (TN) if they are not predicted to be SS and False Negatives (FN) otherwise. To evaluate the performance of the $\mathrm{CNN}$ models, five metrics were used:

Accuracy is the ratio of the number of correct predictions to the total number of predictions:

$$
\text { Accuracy }=\frac{(T P+T N)}{(T P+T N+F P+F N)}
$$

Precision is the ratio of the number of correctly predicted SS to the total number of predicted SS:

$$
\text { Precision }=\frac{T P}{(T P+F P)}
$$

Sensitivity (also known as recall) is the ratio of the number of correctly predicted SS to the total number of SS:

$$
\text { Sensitivity }=\frac{T P}{(T P+F N)}
$$

Specificity is the ratio of the number of correctly predicted non-SS sequences to the total number of non-SS sequences:

$$
\text { Specificity }=\frac{T N}{(T N+F P)}
$$

F1 Score is the harmonic mean of the precision and sensitivity and shows a balance between these two metrics:

$$
F 1 \text { score }=2 * \frac{\text { Precision } * \text { Sensitivity }}{\text { Precision }+ \text { Sensitivity }}
$$

\section{Independent benchmarks of SS from model and non-model organisms}

To estimate the reliability and robustness of the CNN models, they were evaluated on 5 large-scale benchmarks, including sequences from: Human, D. rerio (Fish), D. melanogaster (Fly), C. elegans (Worm) and A. thaliana (Plant), that were downloaded from https://public.bmi.inf.ethz.ch/user/behr/splicing/ [31]. A selection of 10,000 SS and 10,000 non-SS sequences was performed for each benchmark, including a number of non-canonical SS (human: 307; fish: 85; fly:120; worm: 67 and plant: 122).

To evaluate the performance of the models on non-model organisms, we constructed one other independent benchmark called 'PVP' (Protist and ViridiPlantae), containing sequences from protists and viridiplantae. The sequence selection process is similar to that used in G3PO. The reference sequences are the cytoplasmic tryptophanyl-tRNA synthetase of Paramecium tetraurelia (A0D783_PARTE) and the tryptophan-tRNA ligase of Arabidopsis thaliana (SYWM_ARATH). All orthologs 
were extracted from the OrthoInspector database version 3 [74], multiple sequence alignments were obtained with PipeAlign version 2 [80], and manually analyzed to identify 62 'Confirmed' sequences (33 plants and 29 protists). Finally, the benchmark contains 692 (with 21 non-canonical) donor SS and 714 (with 18 non-canonical) acceptor SS, and the same number of non-SS sequences to balance the dataset.

The benchmarks were used to compare Spliceator with other existing SS prediction methods, including NNSplice, MaxEntScan, DSSP and SpliceFinder. Note that SpliceRover was not included in these large-scale benchmark tests, since the method is only available as a web server. Moreover, for a fair evaluation, only tools using the raw DNA sequences as features were included. Hence, SpliceAI was also not selected because the input file must be in Variant Call Format (.vcf) and not a raw sequence. In addition, SpliceAI models were trained with GENCODE data coupled with experimental data.

\section{Explicability}

The visualization heatmaps of the nucleotides most used by the models were generated using the Grad-CAM (Gradient Class Activation Map) technique [81]. The maps were generated from the training sets: the higher the score, the warmer the color (yellow) and the lower the score, the colder the color (deep blue). In order to highlight the most representative patterns identified during the training process, the heatmaps were averaged from 10,000 samples for each class of each SS.

\section{Abbreviations}

NGS: Next generation sequencing; RNA-seq: MRNA-sequencing; UTR: UnTranslated Region; SS: Splice Site; ML: Machine Learning; DL: Deep Learning; CNN: Convolutional Neural Network; BPS: BranchPoint site; ISE: Intronic Splicing Enhancer; ISS: Intronic Splicing Silencer; ESE: Exonic Splicing Enhancer; ESS: Exonic Splicing Silencer; FP: False Positive; AS: All Sequences; GS: Gold Standard; nt: Nucleotide; PPT: PolyPyrimidine Tract; PVP: Protists and ViridiPlantea; BBS: Bardet-Biedl Syndrome; ReLU: Rectified Linear Unit; TP: True Positive; FP: False Positive; TN: True Negative; FN: False Negative.

\section{Supplementary Information}

The online version contains supplementary material available at https://doi.org/10.1186/s12859-021-04471-3.

Additional file 1. Figures S1-S2 and Tables S1-S6

Additional file 2. Tables S1-S3, performance results of donor and acceptor models

\section{Acknowledgements}

The authors would like to thank the BiGEst-ICube bioinformatics platform for assistance.

\section{Authors' contributions}

NS, OP and JDT conceived the study and designed analyses. AJG and PC contributed to designing and implementing the CNN method. NS and OP generated and curated training and test datasets. NS performed predictions, evaluations and benchmarks, with support from AK, RO, TW and LM. NS, OP and JDT analyzed the data, with input from all authors. NS, AJG and JDT wrote the manuscript, with input from all authors. All authors read and approved the final manuscript.

\section{Funding}

This work was supported by French Infrastructure Institut Français de Bioinformatique (IFB) ANR-11-INBS-0013, and the ANR projects Elixir-Excelerate: GA-676559 and RAinRARE: ANR-18-RAR3-0006-02, and Institute funds from the French Centre National de la Recherche Scientifique, the University of Strasbourg. The funding bodies played no role in the design of the study and collection, analysis, and interpretation of data or in writing the manuscript.

\section{Availability of data and materials}

Spliceator source code (python) and the datasets analyzed or generated are available at: https://git.unistra.fr/nscalzitti/ spliceator, and a web service is available at: www.lbgi.fr/spliceator/. 


\section{Declarations}

Ethics approval and consent to participate

Not applicable.

\section{Consent for publication}

Not applicable.

\section{Competing interests}

JDT is a member of the editorial board (Associate Editor) of this journal. The authors declare they have no other competing interests.

\section{Author details}

${ }^{1}$ Complex Systems and Translational Bioinformatics (CSTB), ICube Laboratory, UMR7357, University of Strasbourg, 1 rue Eugène Boeckel, 67000 Strasbourg, France. ${ }^{2}$ BiGEst-ICube Platform, ICube Laboratory, UMR7357, 1 rue Eugène Boeckel, 67000 Strasbourg, France.

Received: 4 July 2021 Accepted: 9 November 2021

Published online: 23 November 2021

\section{References}

1. Brůna T, Hoff KJ, Lomsadze A, Stanke M, Borodovsky M. BRAKER2: automatic eukaryotic genome annotation with GeneMark-EP+ and AUGUSTUS supported by a protein database. bioRxiv. Cold Spring Harbor Laboratory; 2020;2020.08.10.245134.

2. Campbell MS, Holt C, Moore B, Yandell M. Genome annotation and curation using MAKER and MAKER-P. Curr Protoc Bioinformatics. 2014;48:4.11.1-4.11.39.

3. Haas BJ, Delcher AL, Mount SM, Wortman JR, Smith RK Jr, Hannick LI, et al. Improving the Arabidopsis genome annotation using maximal transcript alignment assemblies. Nucleic Acids Res. 2003;31:5654-66.

4. Yates AD, Achuthan P, Akanni W, Allen J, Allen J, Alvarez-Jarreta J, et al. Ensembl 2020. Nucleic Acids Res. 2020:48:D682-8.

5. Thibaud-Nissen F, DiCuccio M, Hlavina W, Kimchi A, Kitts PA, Murphy TD, et al. P8008 The NCBl eukaryotic genome annotation pipeline. J Anim Sci. 2016:94:184-184.

6. Stanke M, Schöffmann O, Morgenstern B, Waack S. Gene prediction in eukaryotes with a generalized hidden Markov model that uses hints from external sources. BMC Bioinformatics. 2006;7:62.

7. Burge C, Karlin S. Prediction of complete gene structures in human genomic DNA. J Mol Biol. 1997;268:78-94.

8. Korf I. Gene finding in novel genomes. BMC Bioinform. 2004;4:1-9.

9. Majoros WH, Pertea M, Salzberg SL. TigrScan and GlimmerHMM: two open source ab initio eukaryotic gene-finders. Bioinformatics. 2004:20:2878-9.

10. Yandell M, Ence D. A beginner's guide to eukaryotic genome annotation. Nat Rev Genet. 2012;13:329-42.

11. Salzberg SL. Next-generation genome annotation: we still struggle to get it right. Genome Biol. 2019;20:92. https:// doi.org/10.1186/s13059-019-1715-2.

12. Meyer C, Scalzitti N, Jeannin-Girardon A, Collet P, Poch O, Thompson JD. Understanding the causes of errors in eukaryotic protein-coding gene prediction: a case study of primate proteomes. BMC Bioinformatics. 2020;21:513.

13. Zhang D, Guelfi S, Garcia-Ruiz S, Costa B, Reynolds RH, D'Sa K, et al. Incomplete annotation has a disproportionate impact on our understanding of Mendelian and complex neurogenetic disorders. Sci Adv. 2020;6:eaay8299.

14. Rogozin IB, Carmel L, Csuros M, Koonin EV. Origin and evolution of spliceosomal introns. Biol Direct. 2012;7:11.

15. Matera AG, Wang Z. A day in the life of the spliceosome. Nat Rev Mol Cell Biol. 2014;15:108-21.

16. Pan Q, Shai O, Lee LJ, Frey BJ, Blencowe BJ. Deep surveying of alternative splicing complexity in the human transcriptome by high-throughput sequencing. Nat Genet. 2008:40:1413-5.

17. Ben-Dov C, Hartmann B, Lundgren J, Valcárcel J. Genome-wide analysis of alternative pre-mRNA splicing. J Biol Chem. 2008:283:1229-33.

18. Burset M, Seledtsov IA, Solovyev W. SpliceDB: database of canonical and non-canonical mammalian splice sites. Nucleic Acids Res. 2001:29:255-9.

19. Nguyen H, Das U, Wang B, Xie J. The matrices and constraints of GT/AG splice sites of more than 1000 species/lineages. Gene. 2018;660:92-101.

20. Burset M, Seledtsov IA, Solovyev W. Analysis of canonical and non-canonical splice sites in mammalian genomes. Nucleic Acids Res. 2000;28:4364-75.

21. Frey K, Pucker B. Animal, fungi, and plant genome sequences harbor different non-canonical splice sites. Cells. 2020;9:458.

22. Sheth N, Roca X, Hastings ML, Roeder T, Krainer AR, Sachidanandam R. Comprehensive splice-site analysis using comparative genomics. Nucleic Acids Res. 2006;34:3955-67.

23. Pucker B, Brockington SF. Genome-wide analyses supported by RNA-Seq reveal non-canonical splice sites in plant genomes. BMC Genomics. 2018;19:980.

24. Pucker B, Holtgräwe D, Weisshaar B. Consideration of non-canonical splice sites improves gene prediction on the Arabidopsis thaliana Niederzenz-1 genome sequence. BMC Res Notes. 2017;10:667.

25. Wang K, Singh D, Zeng Z, Coleman SJ, Huang Y, Savich GL, et al. MapSplice: accurate mapping of RNA-seq reads for splice junction discovery. Nucleic Acids Res. 2010;38:e178-e178.

26. Trapnell C, Pachter L, Salzberg SL. TopHat: discovering splice junctions with RNA-Seq. Bioinformatics. 2009:25:1105-11. 
27. Ameur A, Wetterbom A, Feuk L, Gyllensten U. Global and unbiased detection of splice junctions from RNA-seq data. Genome Biol. 2010;11:R34.

28. Ozsolak F, Milos PM. RNA sequencing: advances, challenges and opportunities. Nat Rev Genet. 2011;12:87-98.

29. Degroeve S, De Baets B, Van de Peer Y, Rouzé P. Feature subset selection for splice site prediction. Bioinformatics. 2002;18(Suppl 2):S75-83.

30. Degroeve S, Saeys Y, De Baets B, Rouzé P, Van de Peer Y. SpliceMachine: predicting splice sites from high-dimensional local context representations. Bioinformatics. 2005;21:1332-8.

31. Sonnenburg S, Schweikert G, Philips P, Behr J, Rätsch G. Accurate splice site prediction using support vector machines. BMC Bioinformatics. 2007:8:57.

32. Maji S, Garg D. Hybrid approach using SVM and MM2 in splice site junction identification. Curr Bioinform. 2014;9:76-85.

33. Pashaei E, Yilmaz A, Ozen M, Aydin N. A novel method for splice sites prediction using sequence component and hidden Markov model. Annu Int Conf IEEE Eng Med Biol Soc. 2016;2016:3076-9.

34. Zhang Q, Peng Q, Zhang Q, Yan Y, Li K, Li J. Splice sites prediction of Human genome using length-variable Markov model and feature selection. Expert Syst Appl. 2010;37:2771-82.

35. Pashaei E, Ozen M, Aydin N. Splice site identification in human genome using random forest. Heal Technol. 2016;1:141-52.

36. Meher PK, Sahu TK, Rao AR. Prediction of donor splice sites using random forest with a new sequence encoding approach. BioData Mining. 2016;9:4.

37. Chen T-M, Lu C-C, Li W-H. Prediction of splice sites with dependency graphs and their expanded bayesian networks. Bioinformatics. 2005;21:471-82.

38. Saeys $Y$, Degroeve $S$, Van de Peer $Y$. Digging into acceptor splice site prediction: an iterative feature selection approach. In: Boulicaut J-F, Esposito F, Giannotti F, Pedreschi D, editors. Knowledge discovery in databases: PKDD 2004. Berlin, Heidelberg: Springer; 2004. p. 386-97.

39. Naito T. Human splice-site prediction with deep neural networks. J Comput Biol. 2018;25:954-61.

40. Zuallaert J, Godin F, Kim M, Soete A, Saeys Y, De Neve W. SpliceRover: interpretable convolutional neural networks for improved splice site prediction. Bioinformatics. 2018;34:4180-8.

41. Wang R, Wang Z, Wang J, Li S. SpliceFinder: ab initio prediction of splice sites using convolutional neural network. BMC Bioinformatics. 2019;20:652.

42. Jaganathan K, Kyriazopoulou Panagiotopoulou S, McRae JF, Darbandi SF, Knowles D, Li Yl, et al. Predicting Splicing from Primary Sequence with Deep Learning. Cell. 2019;176:535-548.e24.

43. LeCun Y, Bengio Y, Hinton G. Deep learning. Nature. 2015;521:436-44.

44. Li Y, Huang C, Ding L, Li Z, Pan Y, Gao X. Deep learning in bioinformatics: Introduction, application, and perspective in the big data era. Methods. 2019;166:4-21.

45. Scalzitti N, Jeannin-Girardon A, Collet P, Poch O, Thompson JD. A benchmark study of ab initio gene prediction methods in diverse eukaryotic organisms. BMC Genomics. 2020;21:293.

46. Kilkenny MF, Robinson KM. Data quality: "Garbage in - garbage out." Health Inf Manag. 2018;47:103-5.

47. Reese MG, Eeckman FH, Kulp D, Haussler D. Improved splice site detection in genie. J Comput Biol. 1997;4:311-23.

48. Yeo G, Burge CB. Maximum entropy modeling of short sequence motifs with applications to RNA splicing signals. J Comput Biol. 2004;11:377-94.

49. Cartegni L, Chew SL, Krainer AR. Listening to silence and understanding nonsense: exonic mutations that affect splicing. Nat Rev Genet. 2002;3:285-98.

50. Zeng Y, Yuan H, Yuan Z, Chen Y. A high-performance approach for predicting donor splice sites based on short window size and imbalanced large samples. Biol Direct. 2019;14:6.

51. Mercer TR, Clark MB, Andersen SB, Brunck ME, Haerty W, Crawford J, et al. Genome-wide discovery of human splicing branchpoints. Genome Res. 2015;25:290-303.

52. Anna A, Monika G. Splicing mutations in human genetic disorders: examples, detection, and confirmation. J Appl Genet. 2018;59:253-68.

53. Gooding C, Clark F, Wollerton MC, Grellscheid S-N, Groom H, Smith CW. A class of human exons with predicted distant branch points revealed by analysis of AG dinucleotide exclusion zones. Genome Biol. 2006;7:R1.

54. Campbell M, Hoane AJ, Hsu F. Deep blue. Artif Intell. 2002;134:57-83.

55. Silver D, Huang A, Maddison CJ, Guez A, Sifre L, van den Driessche G, et al. Mastering the game of Go with deep neural networks and tree search. Nature. 2016;529:484-9.

56. AlQuraishi M. AlphaFold at CASP13. Bioinformatics. 2019;35:4862-5.

57. Senior AW, Evans R, Jumper J, Kirkpatrick J, Sifre L, Green T, et al. Improved protein structure prediction using potentials from deep learning. Nature. 2020;577:706-10.

58. Li H, Tian S, Li Y, Fang Q, Tan R, Pan Y, et al. Modern deep learning in bioinformatics. J Mol Cell Biol. 2020;12:823-7.

59. Koumakis L. Deep learning models in genomics; are we there yet? Comput Struct Biotechnol J. 2020;18:1466-73.

60. Tang B, Pan Z, Yin K, Khateeb A. Recent Advances of Deep Learning in Bioinformatics and Computational Biology. Front Genet. 2019;10:214. https://doi.org/10.3389/fgene.2019.00214.

61. HeY, Shen Z, Zhang Q, Wang S, Huang DS. A survey on deep learning in DNA/RNA motif mining. Brief Bioinform. 2021. https://doi.org/10.1093/bib/bbaa229.

62. Krizhevsky A, Sutskever I, Hinton GE. ImageNet classification with deep convolutional neural networks. Commun ACM. 2017;60:84-90.

63. Szegedy C, Liu W, Jia Y, Sermanet P, Reed S, Anguelov D, et al. Going Deeper with Convolutions. arXiv:1409.4842 [cs]. 2014 [cited 2021 Jan 6].

64. Pollastro P, Rampone S. Hs3d, a dataset of homo sapiens splice regions, and its extraction procedure from a major public database. Int J Mod Phys C. 2002;13:1105-17.

65. Pertea M, Lin X, Salzberg SL. GeneSplicer: a new computational method for splice site prediction. Nucleic Acids Res. 2001;29:1185-90. 
66. Khodabandelou G, Routhier E, Mozziconacci J. Genome annotation across species using deep convolutional neural networks. PeerJ Comput Sci. 2020;6:e278.

67. Stiehler F, Steinborn M, Scholz S, Dey D, Weber APM, Denton AK. Helixer: cross-species gene annotation of large eukaryotic genomes using deep learning. Bioinformatics. 2020;36:5291-8.

68. The UniProt Consortium. UniProt: the universal protein knowledgebase in 2021. Nucleic Acids Res. 2021;49:D480-9.

69. Tørresen OK, Star B, Mier P, Andrade-Navarro MA, Bateman A, Jarnot P, et al. Tandem repeats lead to sequence assembly errors and impose multi-level challenges for genome and protein databases. Nucleic Acids Res. 2019:47:10994-1006.

70. Zhang C, Li W-H, Krainer AR, Zhang MQ. RNA landscape of evolution for optimal exon and intron discrimination PNAS Natl Acad Sci. 2008;105:5797-802.

71. Gao K, Masuda A, Matsuura T, Ohno K. Human branch point consensus sequence is yUnAy. Nucleic Acids Res. 2008;36:2257-67.

72. Soemedi R, Cygan KJ, Rhine C, Glidden DT, Taggart AJ, Lin C-L, et al. The Effects of Structure on pre-mRNA processing and stability. Methods. 2017;125:36-44.

73. Tellier M, Maudlin I, Murphy S. Transcription and splicing: a two-way street. WIREs RNA. 2020;11:e1593.

74. Nevers Y, Kress A, Defosset A, Ripp R, Linard B, Thompson JD, et al. Ortholnspector 3.0: open portal for comparative genomics. Nucleic Acids Res. 2019;47:D411-8.

75. Crooks GE, Hon G, Chandonia J-M, Brenner SE. WebLogo: a sequence logo generator. Genome Res. 2004;14:1188-90.

76. Abadi M, Barham P, Chen J, Chen Z, Davis A, Dean J, et al. TensorFlow: A system for large-scale machine learning. arXiv:1605.08695 [cs]. 2016 [cited 2021 Jan 6].

77. Pedregosa F, Varoquaux G, Gramfort A, Michel V, Thirion B, Grisel O, et al. Scikit-learn: machine learning in Python. J Mach Learn Res. 2011;12:2825-30.

78. Srivastava N, Hinton G, Krizhevsky A, Sutskever I, Salakhutdinov R. Dropout: a simple way to prevent neural networks from overfitting. J Mach Learn Res. 2014;15:1929-58.

79. Kingma DP, Ba J. Adam: A Method for Stochastic Optimization. arXiv:1412.6980 [cs]. 2017 [cited 2021 Jan 6].

80. Plewniak F, Bianchetti L, Brelivet Y, Carles A, Chalmel F, Lecompte O, et al. PipeAlign: a new toolkit for protein family analysis. Nucleic Acids Res. 2003;31:3829-32.

81. Selvaraju RR, Cogswell M, Das A, Vedantam R, Parikh D, Batra D. Grad-CAM: visual explanations from deep networks via gradient-based localization. Int J Comput Vis. 2020;128:336-59.

\section{Publisher's Note}

Springer Nature remains neutral with regard to jurisdictional claims in published maps and institutional affiliations.

- fast, convenient online submission

- thorough peer review by experienced researchers in your field

- rapid publication on acceptance

- support for research data, including large and complex data types

- gold Open Access which fosters wider collaboration and increased citations

- maximum visibility for your research: over 100M website views per year

At BMC, research is always in progress.

Learn more biomedcentral.com/submissions 